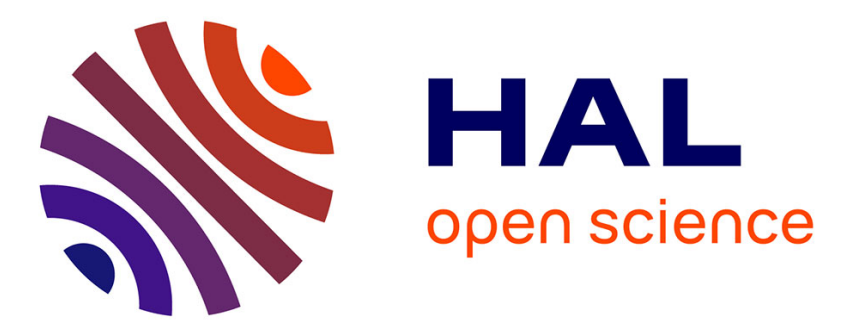

\title{
Dripping and jetting of semi-dilute polymer solutions co-flowing in co-axial capillaries
}

S A Vagner, S A Patlazhan, C A Serra, D Funfschilling, V G Kulichikhin

\section{To cite this version:}

S A Vagner, S A Patlazhan, C A Serra, D Funfschilling, V G Kulichikhin. Dripping and jetting of semi-dilute polymer solutions co-flowing in co-axial capillaries. Physics of Fluids, 2021. hal-03422948

\section{HAL Id: hal-03422948 \\ https://hal.science/hal-03422948}

Submitted on 9 Nov 2021

HAL is a multi-disciplinary open access archive for the deposit and dissemination of scientific research documents, whether they are published or not. The documents may come from teaching and research institutions in France or abroad, or from public or private research centers.
L'archive ouverte pluridisciplinaire HAL, est destinée au dépôt et à la diffusion de documents scientifiques de niveau recherche, publiés ou non, émanant des établissements d'enseignement et de recherche français ou étrangers, des laboratoires publics ou privés. 


\title{
Dripping and jetting of semi-dilute polymer solutions co-flowing in co-axial capillaries
}

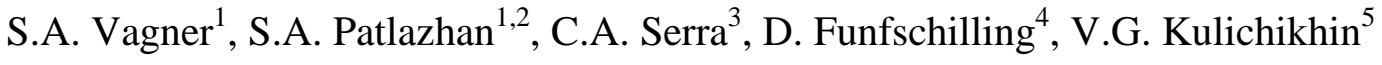 \\ ${ }^{1}$ Institute of Problems of Chemical Physics, Russian Academy of Sciences, Academician \\ Semenov Avenue 1, Chernogolovka, Moscow Region 142432, Russia \\ ${ }^{2}$ N.N. Semenov Federal Research Center for Chemical Physics, Russian Academy of Sciences, \\ Kosygin Street 4, Moscow 119991, Russia \\ ${ }^{3}$ Université de Strasbourg, CNRS, Institut Charles Sadron UPR 22, F-67000 Strasbourg, France \\ ${ }^{4}$ Université de Strasbourg, CNRS, ICube UMR 7357, 2, rue Boussingault, F-67000 Strasbourg, \\ France
}

${ }^{5}$ A.V. Topchiev Institute of Petrochemical Synthesis, Russian Academy of Sciences, 29, Leninsky Avenue, Moscow 119991, Russia,

\begin{abstract}
In this work, the mechanisms of dripping and jetting modes of semi-dilute polyacrylamide aqueous solutions co-flowing along with silicone oil in coaxial capillaries were studied. In the dripping mode, the elongation rate of the liquid filament between the droplet and the inner capillary is controlled by the continuous medium flow rate and does not depend on polymer concentration. The diameter of the filament decreases both by increasing its length and due to the squeezing-out of the polymer solution into the droplet under the influence of capillary pressure. In the jetting mode, the liquid thread diameter was found to evolve in three stages. In the first stage, the average jet velocity increases, whereas in the second and third stages, the jet spreads at a permanent rate corresponding to the maximum flow velocity of the continuous medium. The transition from the second to the third stage is defined by the appearance of capillary waves leading to formation of liquid beads on the string. In the third stage, the diameter of filament between the beads decreases exponentially and is governed by the relaxation time which is strongly dependent on polymer concentration but independent on the silicon oil continuous phase flow rate. The evolution of dimensions of the liquid filaments and beads was investigated. It was found that the filament length between beads decreases as a power law function of time. Also, the evolution of the transverse dimensions of filaments and beads with the development of jet capillary instability has a universal character independent of the polymer concentration and continuous medium flow rates.
\end{abstract}




\section{Introduction}

The development of capillary instabilities in the dripping and jetting modes in flow of polymer solution differs significantly from those of Newtonian liquid ${ }^{1,2}$. Indeed, if a low molecular liquid thread breaks up quickly due to the Rayleigh-Plateau instability ${ }^{3-6}$, the addition of a small amount of polymer leads to a significant alteration of the mechanism of development of dispersed phase instability: in a dripping mode, the droplet formed remains connected with its source through a long filament while in the jetting mode, jets disintegrate into a set of liquid beads interconnected by thin filaments ("beads-on-string") remaining stable for relatively long times [7-15; 2017ZhuWang_rev]. Formation of long filaments is a strongly nonlinear dynamical process despite the fact that the classical linear analysis indicates that a viscoelastic jet should be less stable ${ }^{16}$. This is related to the fact that the stability of filaments of viscoelastic fluids is controlled intimately by capillary, viscous, inertial, and elastic forces ${ }^{12,17-21}$. The addition of a small amount of polymer was shown to stabilize efficiently the filament thinning. Physically, this effect occurs due to the balance of elastic stresses and capillary pressure in the squeezed viscoelastic filament [10, 14, 23, 25,26, J. Eggers, M. A. Herrada, and J. H. Snoeijer, J. Fluid Mech., 887, A19 (2020)]. Eventually, the mean diameter of the filament between neighboring beads decreases exponentially with a rate determined by the relaxation time of the polymer solution $^{22-24}$. In the later stages preceding rupture, the thinned filaments emit much smaller droplets $^{12,15,18,25,26}$. Despite the fact that such a structure appears to resemble a "beads-on-string", it is called as a "blistering" pattern ${ }^{15,25}$ because it is associated with other physical processes governed by the polymer and solvent phase separation ${ }^{26-30}$.

The instability of jets of polymer solution was mainly investigated using three types of experimental setups: (i) separation of two plates wetted by a liquid drop [12,14,23,25], (ii) a jet flowing out of a nozzle ${ }^{31}$, and (iii) drop detachment by gravity [32,33; C. Clasen, J. Bico, V.M. Entov, and G.H. Mckinley, J. Fluid Mech. 636, 5-40 (2009)]. Most of the experiments were carried out in air. The instability of polymeric jets was also observed in a quiescent viscous media just after stopping the droplet stretching in the four-roll mill device. ${ }^{34}$

Along with progresses in microfluidics, studies of formation of monodisperse microdroplets and jets under confinement conditions have acquired new motivations due to their scientific interest as well as for their numerous applications in life science (L. Derzsi, M. Kaspryk, J.-P. Plog, P. Garstecki Physics of Fluids 25, 092001 2013). A thorough understanding of hydrodynamic behavior of two-phase non-Newtonian fluids in microfluidic devices is essential for both fundamental problems and applications [2001StoneKim, 2004StoneStroock, 2005SquiresQuake]. In particular, the influence of the outer conditions during development of capillary instabilities in viscoelastic jets i.e. in a quiescent environment [J.H. Snoeijer, A. 
Pandey, M.A. Herrada, and J. Eggers, Proc. R. Soc. A 476, 20200419 (2020)] or in a flowing medium [XueChen2020, L. Lei, Y. Zhao, W. Chen, H. Li, X. Wang, and J. Zhang, Energies, 14, 1341 (2021)] should be taken into account. In the first case, the filament length is controlled by external forces, while in the second case, the dynamics of the dispersed phase depends on the continuous phase flow conditions as it takes place in microchannels.

The dripping and jetting flow modes were observed for both Newtonian and nonNewtonian dispersed fluids in different microfluidic devices including flow-focusing [2003AnnaBontoux, 2017Sarah, 2019Rahimi, XueChen2020, 36], cross-slot microchannels [35; Arratia2009], microfluidic T-junction [Q. Zhang, C. Zhu, W. Du, C. Liu, T. Fu, Y. Ma, H.Z. Li, Chem. Eng. Res. Design, 139, 188-196 (2018); A. Yagodnitsyna, A. Kovalev, and A. Bilsky, 12, 335 (2021)], co-axial capillaries [2008Serra, 2014WuChen; 2015LanLiLuo; S.Yi. Mak, Y. Chao, and H.C. Shum, RSC Adv. 7, 3287-3292 (2017); 2017DengWangHuang]. However, the hydrodynamic conditions for droplet and jet formation are different in these systems. In a flow focusing device, two immiscible fluids converge near a contraction in such a way that in the dripping mode, squeezing of the dispersed phase leads to the formation of droplets, while in the jetting mode, a strong elongation flow occurs in this junction. The similar flow patterns are expected to form in the cross-slot and T-Junction microchannels. On the contrary, due to the axisymmetric flow in coaxial capillaries, the continuous and dispersed phases meet in parallel which simplifies the flow structure, allowing the hydrodynamic instabilities of the dispersed phase to be investigated without additional complications.

For Newtonian fluids, the main difference between dripping and jetting modes is in balance between two time scales: (i) the time scale of the squeezing and development of Rayleigh-Plateau instability and (ii) the time scale of the flow velocity [2015LanLiLuo]. The increase in the mean velocity of the continuous phase results in a decrease of droplet size which then favors the dripping-to-jetting transition [2007Utada, 2013NunesTsai, 2015LanLiLuo, 2017DengWangHuang]. In the case of non-Newtonian viscoelastic fluids, the balance is different because elasticity, as already mentioned, significantly slows down the last moment of thinning of the liquid filament linking the droplet in formation to the capillary. Another important difference between Newtonian and non-Newtonian dispersed phase is that the break-up in the Newtonian case follows a power law decay, which is similar to the early time scale of the viscoelastic nonNewtonian fluid, but then there is a transition to a slower thinning where elastic stress is dominant [2013Derzsi, 2019Dinic; J.H. Snoeijer, A. Pandey, M.A. Herrada, J. Eggers. Proc. R. Soc. A 476, 20200419 (2020)]. It should be noted that most of results were derived for dilute polymer solutions $[22,23,25,25]$. 
In contrast to the quiescent environment, it is expected that flow of two-phase fluid in various microchannel can upset the delicate balance between elastic and capillary forces in the dispersed phase resulting in changes in droplets and jets formation. Since the velocity and pressure fields in flow-focusing and co-flow devices are rather different, the influence of viscoelasticity on the dynamical behaviour of the dispersed phase should be different as well. The previous studies of viscoelastic droplet formation in microchannels were carried out mostly in flow-focusing or cross-slot microfluidic devices [35; Arratia2008, Zhoe2006, du2017, XueChen2020]. In these cases, the polymer solution crosses the orifice and elongates forming a primary droplet connected with a thin filament. Similar effects were observed in a motionless environment. Nevertheless, a number of open questions concerning the physical mechanisms of dripping and jetting flow modes of the non-Newtonian dispersed phase under confinement conditions are of fundamental interest. Especially it concerns dynamical behavior of droplets and jets of semi-dilute polymer solutions in coaxial capillaries, which has not been studied sufficiently.

In this work, we focused on mechanisms of formation of dripping and jetting flow modes for semi-dilute aqueous solutions of long chain flexible polyacrylamide polymer co-flowing with Newtonian silicon oil. Peculiarities of structural evolution and stability of polymeric filaments in the dripping and jetting modes were studied for different polymer concentrations and flow rates of the continuous and dispersed phases.

\section{Experiments}

Flow patterns of a pair of immiscible liquids in coaxial capillaries were studies (Fig. 1). The external capillary with an internal diameter of $d_{c}=1.06 \mathrm{~mm}$ was made of PTFE. The inner capillary was made of silicon dioxide with inner and outer diameters of $d_{\mathrm{d}}^{\text {in }}=150 \mu \mathrm{m}$ and $d_{\mathrm{d}}^{\text {out }}=360 \mu \mathrm{m}$, respectively. The outer capillary was fixed in a transparent solid matrix of crosslinked PDMS (polydimethylsiloxane - Sylgard ${ }^{\mathrm{TM}} 184$ Silicon Elasomer Kit, Dow Silicones), whose refractive index is close to that of PTFE and silicone oil, thus minimizing the lens effect caused by the curved surface of the capillary.

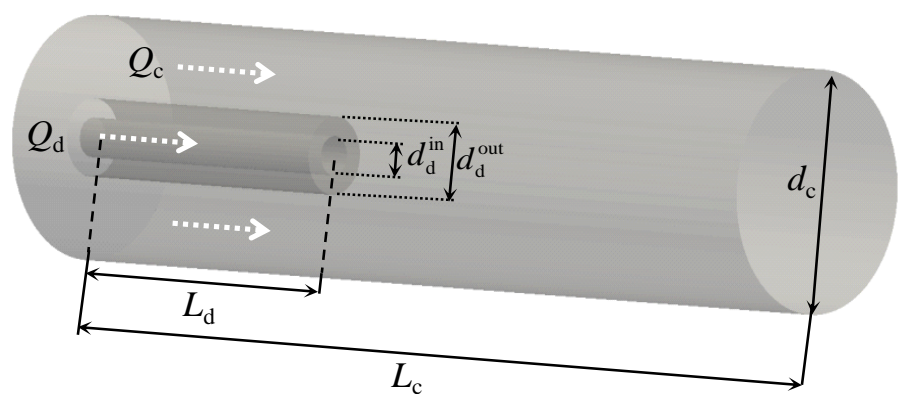


Figure 1. Coaxial capillary microsystem.

Silicone oil of viscosity of $\eta_{c}=0.35 \mathrm{~Pa} \cdot \mathrm{s}$ was fed through the external capillary as the continuous phase, while aqueous solutions of polyacrylamide (PAM) AN $913 \mathrm{SH}$ of the French company SNF Floerger were fed through the inner capillary as the dispersed phase. The anionic PAM has an average molecular weight of $1.2 \cdot 10^{7}$ Dalton. In this case, the critical overlap concentration of the polymer coils is about $c^{*} \cong 0.01 \mathrm{wt} . \%$. Three polymer solutions were studied with PAM concentrations of $c=0.1,0.3$, and 0.6 wt. \% which correspond to semi-dilute solutions with the following ratios $c / c^{*} \cong 10,30$, and 60 , respectively.

Table 1 . The physical parameters for the continuous in dispersed phases at $20^{\circ} \mathrm{C}$.

\begin{tabular}{cccccc}
\hline \hline Physical & \multirow{2}{*}{ Silicon oil } & Distilled & \multicolumn{3}{c}{ PAM aqueous solutions } \\
\cline { 4 - 6 } parameters & & water & 0.1 wt.\% & 0.3 wt.\% & 0.6 wt.\% \\
\hline $\begin{array}{c}\text { Zero-shear-rate } \\
\text { viscosity, Pa·s }\end{array}$ & 0.35 & 0.001 & 8.54 & 47.9 & 146.0 \\
Density, $\mathrm{kg} / \mathrm{m}^{3}$ & 968 & 997 & 998 & 998 & 998 \\
\hline \hline
\end{tabular}

Such fluids belong to non-Newtonian viscoelastic shear thinning fluids which viscosities drops by about three orders of magnitude in the shear rate range from $10^{-3}$ to $100 \mathrm{~s}^{-1}$. Physical parameters of Newtonian silicone oil, distilled water, and PAM aqueous solutions as densities and zero-shear-rate viscosities are presented in Table 1 . The interfacial tension between the dispersed and continuous phases is of $38 \mathrm{mN} / \mathrm{m}$ (measured by N. Kuznetsov) and is independent of the polymer concentration.

The flow rates $Q_{d}$ and $Q_{c}$ of the dispersed and continuous liquid phases were set by means of neMESYS 290N high-precision low-pressure syringe pumps. For the dispersed phase, it was kept constant $Q_{d}=0.005 \mathrm{~mL} / \mathrm{min}$ corresponding to the mean flow velocity of $U_{d}=4.72 \mathrm{~mm} / \mathrm{s}$ in the inner capillary. The silicone oil flow rate was varied from $Q_{c}=0.1$ to $1.0 \mathrm{~mL} / \mathrm{min}$ corresponding to mean flow velocities ranging from $U_{\mathrm{c}}=1.89$ to $18.89 \mathrm{~mm} / \mathrm{s}$. This corresponds to relative flow rates of the continuous phase and dispersed phase in the range $20 \leq Q_{c} / Q_{d} \leq$ 200.

An inverse microscope (Nikon Eclipse TS100) equipped with a 10x/0.25 lens and a Phantom Miro LAB 310 high-speed camera with a resolution of 1280x800 pixels were used to visualize the flow patterns. The frame rate of the camera was kept at $800 \mathrm{fps}$ resulting in an interframe time of $1.25 \mathrm{~ms}$. Such a resolution and image registration rate allowed to investigate in detail the dynamics of dispersed fluid structure formation in a frame of $1.0 \times 1.8 \mathrm{~mm}$. 


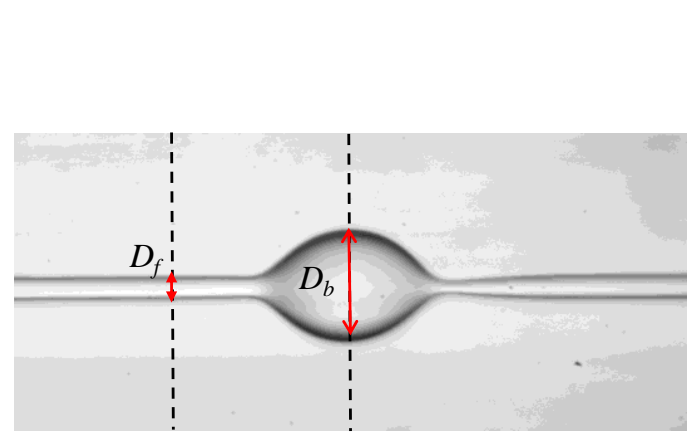

(a)

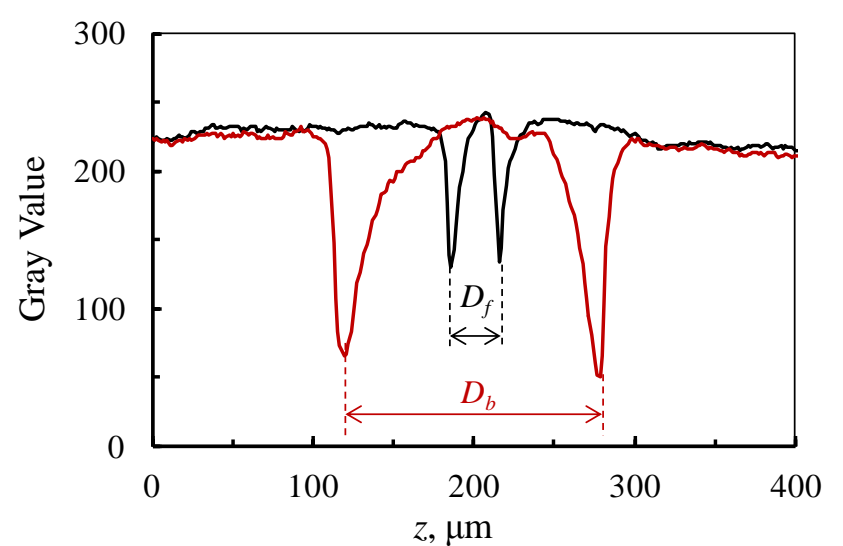

(b)

Figure 3. Fragment of the jet with liquid bead and filaments (a) and level of grey profiles in the cross sections corresponding to the vertical dashed lines (b).

The jet dimensions were calculated using the ImageJ program. Using this program, we plotted the level of grey profiles across different jet sections (the dashed lines in Fig. 3a). Images are coded on 8 bits, that means a zero value corresponds to pure black, while 255 corresponds to pure white. The corresponding grayscale profiles for the filament and bead are presented in Fig. 3b. The minima on these curves correspond to the interface positions along the vertical $z$-axis. The diameter $D_{f}$ of the filament and the maximum transversal size $D_{b}$ of the bead (see Section C3 ) are given by the difference in $z$-coordinates of the curves' minima (see Fig. 2b). To evaluate variations of the jet size over time (one second corresponded to 800 images), a special plug-in was created in the ImageJ program, which resulted in generation of an array of gray value data for all images. This array was processed using a special script in MATLAB ${ }^{\circledR}$, the purpose of which was to calculate the transverse dimensions of the jet for all time moments.

\section{Results and discussion}

\section{A. Flow modes}

The dispersed phase morphology in coaxial capillaries depends on the flow rate ratio between the fluids. The dripping and jetting modes are considered as the reference dynamic states. Before studying the formation of droplets of the PAM aqueous solutions, it first makes sense to consider the corresponding behavior of pure water. Figure 4 shows the formation of water droplets in silicone oil at a flow rate of $Q_{c}=0.1 \mathrm{~mL} / \mathrm{min}$. The droplet formation is preceded by a neck development (see Fig. 4a) whose diameter quickly decreases in the time range from 0.17 to $0.43 \mathrm{~s}$ and rupture at time $t=0.61 \mathrm{~s}$ with the formation of a satellite microdroplet. This mechanism of droplet formation in Newtonian fluids is well known [1-6]. The increase of the continuous phase flow rate up to $1.0 \mathrm{~mL} / \mathrm{min}$ does not lead to fundamental 
changes in the sequence of droplet formation (Fig. 4b-4d), apart from the fact that the droplets become smaller and the necks slightly longer, which is also consistent with the literature $[7,8]$.

(a)

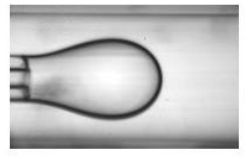

$t=0 \mathrm{~s}$

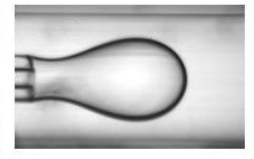

$t=0.17 \mathrm{~s}$

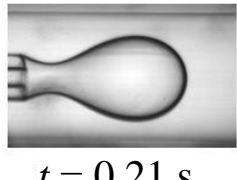

$t=0.21 \mathrm{~s}$

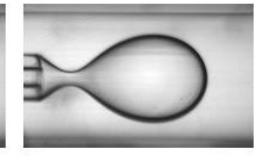

$t=0.25 \mathrm{~s}$

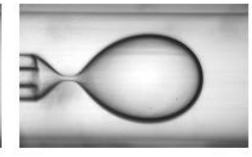

$t=0.43 \mathrm{~s}$

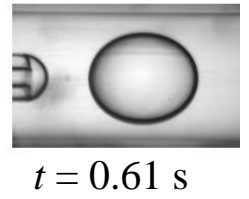

(b)

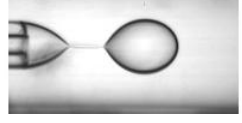

(c)

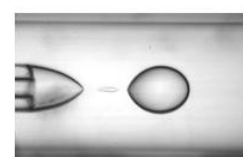

(d)

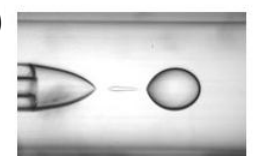

Figure 4. Formation of water droplet in co-flow microsystem with silicone oil as continuous phase with flow rates of respectively $Q_{\mathrm{c}}=0.1$ (a) 0.6 (b), 0.8 (c), and $1.0 \mathrm{~mL} / \mathrm{min}$ (d). The water flow rate was equal to $Q_{\mathrm{d}}=0.005 \mathrm{~mL} / \mathrm{min}$.

The addition of a small amount of polyacrylamide leads to a considerable change in flow patterns. Figure 5a shows that large droplets of PAM aqueous solution of concentration of 0.1 wt.\% were formed in the silicon oil continuous phase at a low flow rate, of $Q_{c}=0.1 \mathrm{~mL} / \mathrm{min}$. As in the case of the water droplet, the neck formation was observed during the time range 0 to 0.21 s. However, after this step, the neck does not disintegrate, but turns into a highly extended cylindrical filament (time range $0.25-0.43 \mathrm{~s}$ ). It loses its stability and ruptures at time $t=0.61 \mathrm{~s}$ with the formation of a long sequence of satellite microdroplets. The increase in silicone oil flow rate leads to some important differences: at $Q_{c}=0.6 \mathrm{~mL} / \mathrm{min}$ newly formed droplets at the exit of the inner capillary remain connected with previously formed droplets. As a result, the formation of several droplets connected by thin filaments was observed (Fig. 5b). The increase in the continuous phase flow rate up to $Q_{c}=0.8$ and $1.0 \mathrm{~mL} / \mathrm{min}$ results in formation of long cylindrical threads (see Fig. 5c and 5d).

(a)

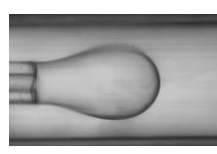

$t=0 \mathrm{~s}$

(b)

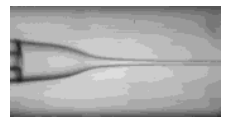

$t=0 \mathrm{~s}$

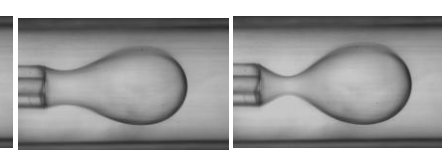

$t=0.17 \mathrm{~s} \quad t=0.21 \mathrm{~s}$

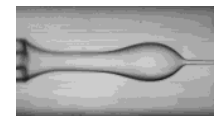

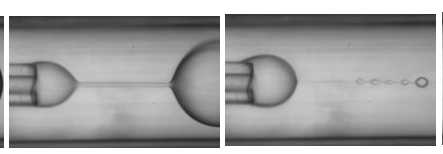

$t=0.43 \mathrm{~s}$

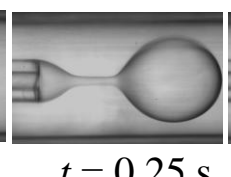

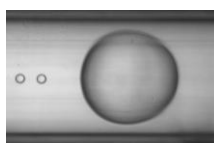

$t=0.61 \mathrm{~s}$

(c)

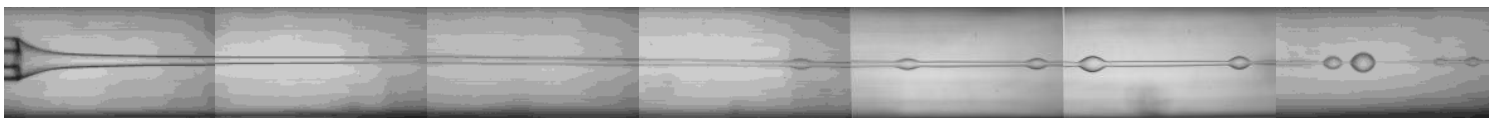

Figure 5. Structures formed by 0.1 wt. $\%$ PAM aqueous solution dispersed phase in co-flow microsystem with silicone oil as continuous phase at different flow rates $Q_{\mathrm{c}}=0.1$ (a), 0.6 (b), 0.8 (c) $\mathrm{mL} / \mathrm{min}$. The PAM aqueous solution flow rate was equal to $Q_{\mathrm{d}}=0.005 \mathrm{~mL} / \mathrm{min}$. 
Thus, depending on flow rate ratios of the dispersed and continuous phases in coaxial capillaries microsystem, different structures of the dispersed phase can be obtained: the dripping, jetting, and intermediate flow modes. In the system under consideration, all flow modes observed differ significantly from those occurring in Newtonian two-phase systems. In the dripping flow mode, the polymer solution forms a long filament linking the inner capillary tip with the terminal droplet. In the jetting flow mode, the polymer solution is pulled into a long thread on which liquid beads are emitted over time due to the capillary instability. In the intermediate mode, the dispersed phase forms a liquid thread as long as in the jetting mode, but possesses a terminal droplet as in the dripping mode. In contrast, pure water forms fairly short jets with small droplets separating from their tips [2-4].

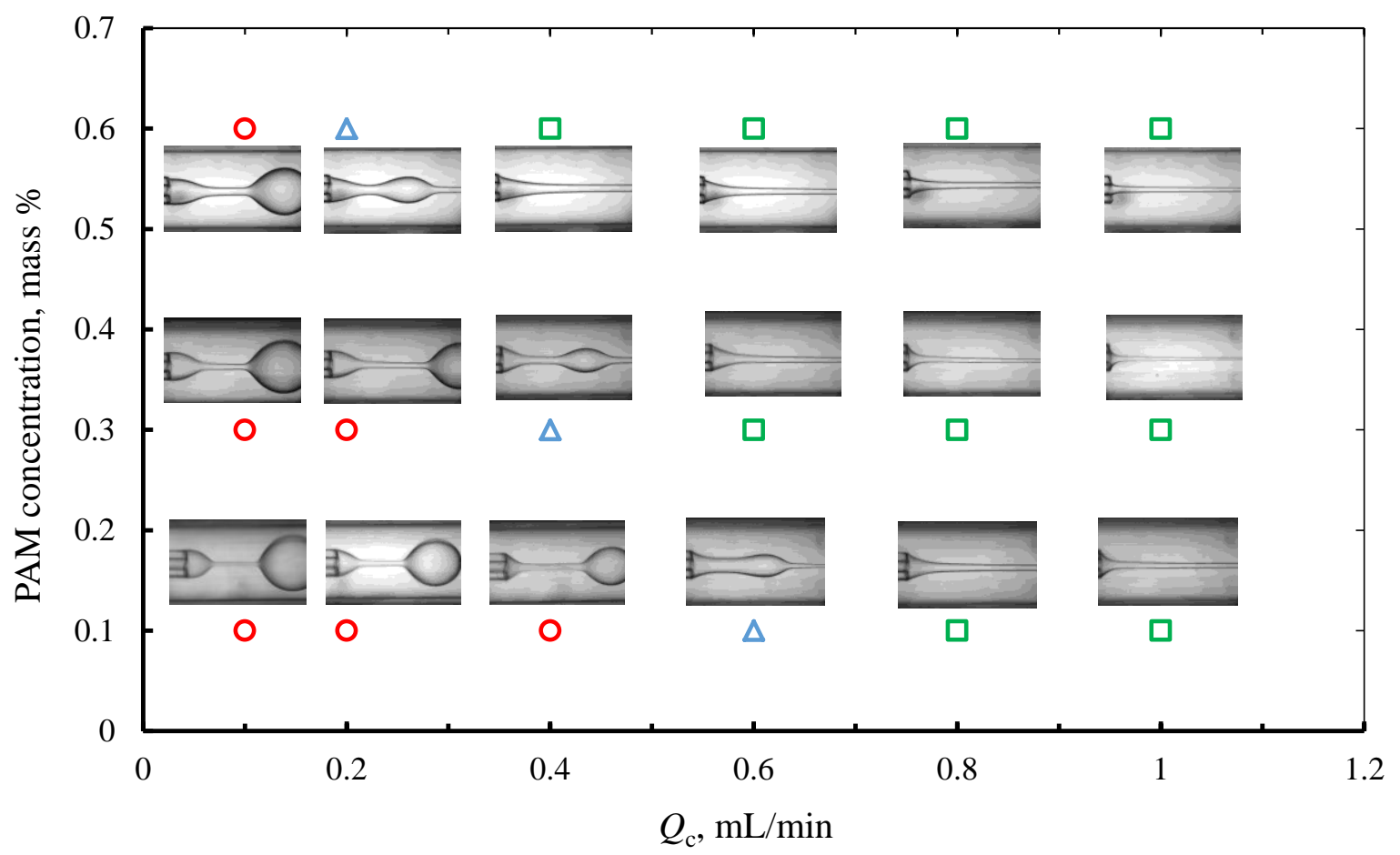

Figure 6. Flow regimes diagram versus PAM aqueous solution concentration and silicone oil flow rate: dripping regime (circle symbols), intermediate regime (triangles), and jetting regime (squares).

The dependence of the observed flow modes on PAM concentration and ratio $Q_{c} / Q_{d}$ of flow rates of the continuous phase and dispersed phases is shown on the diagram presented in Fig. 6. It is seen that increase in the polymer concentration significantly affects the dispersed phase morphology leading to dripping-to-jetting transition at lower flow rate ratios, while increase in the relative flow rate of the continuous phase promotes an earlier onset of jetting mode. In particular, at PAM concentration of $0.1 \mathrm{wt} . \%$, a stable jet is formed at a flow rate ratio of 160 , whereas at $0.6 \mathrm{wt} . \%$ a jet appears at $Q_{c} / Q_{d}=80$. 
Figure 6 shows that increasing the polymer concentration results in smaller droplets and thicker filaments. This is a consequence of a significant increase the viscosity plateau (see Section II) and elastic response of the semi-diluted polymer solutions. It may be a reason why the dripping-to-jetting transition is observed for lower flow rates of the continuous medium as shownin the presented diagram. In the next Sections we carefully study the new peculiarities of the dripping and jetting flow modes of semi-dilute polymer solutions co-flowing with the Newtonian continuous phase in coaxial capillaries at different flow rates.

\section{B. Dripping}

As noted, the detachment of a droplet of polymer solution is preceded by the development of a neck and follows the formation of a long filament connecting the droplet to the inner capillary. The solid curve in Figure 7 shows the evolution of the neck diameter $D_{f}$ of 0.1 wt.\% PAM aqueous solution divided by the outer diameter $d_{d}^{\text {out }}$ of the inner capillary for a continuous phase flow rate of $Q_{c}=0.1 \mathrm{~mL} / \mathrm{min}$. As can be seen, the entire process can be separated into three stages: 1) a slow decrease of the neck diameter, 2) an abrupt neck thinning, and 3) a neck stretching resulting in formation of a long filament due to the motion of the terminal droplet. For the distilled water, the third stage is not at all observed and droplet detachment occurs via rupture of the short neck (see the dashed line in Fig. 7). In the latter case, the neck thinning rate during the second stage is considerably larger than the one for polymer solutions.

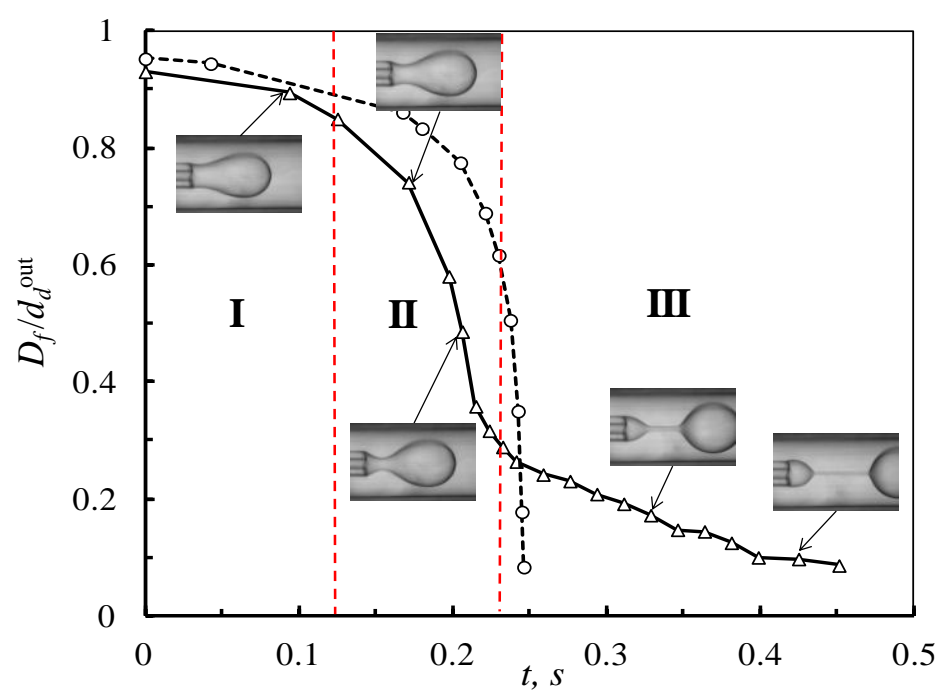

Figure 7. Evolution of the dimensionless neck diameter $D_{f} / d_{d}^{\text {out }}$ for 0.1 wt.\% PAM aqueous solution (solid curve) and distilled water (dashed curve) at continuous and dispersed phase flow rates $Q_{c}=0.1$ and $Q_{d}=0.005 \mathrm{~mL} / \mathrm{min}$, respectively. 


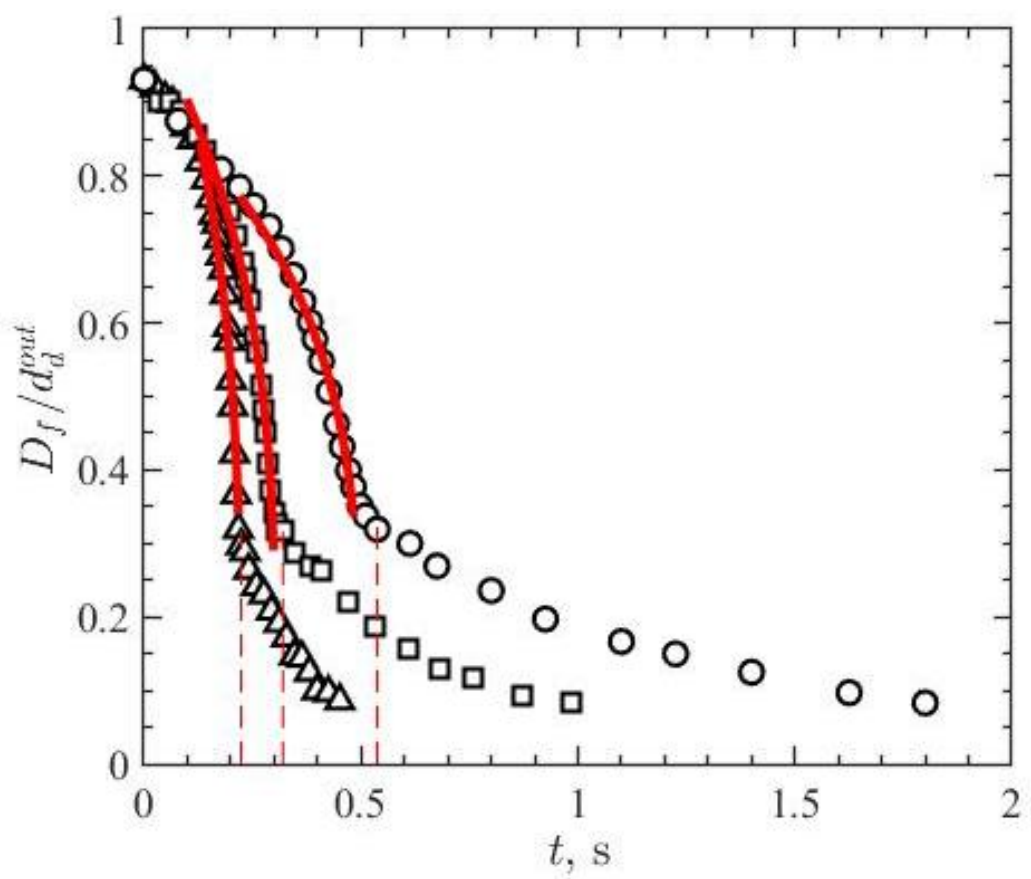

Figure 8. Evolution of the dimensionless neck diameter of PAM aqueous solutions at different polymer concentrations: $c=0.1$ (triangle symbols), 0.3 (squares), and 0.6 wt.\% (circles) in a silicone oil. Flow rates of continuous and dispersed phases are equal to $Q_{c}=0.1$ and $Q_{d}=0.005$ $\mathrm{mL} / \mathrm{min}$, respectively. The red solid lines correspond to the relationship $D_{f} \sim\left(t_{0}-t\right)^{\alpha}$. The dashed lines indicate the onsets $t_{0}$ of the formation of the filaments.

Figure 8 shows that an increase in polymer concentration results in slower neck thinning during the second and third stages of the process. The onset of the formation of a filament as well as its eventual rupture and droplet release, was delayed for higher polymer concentrations. The corresponding onset time $t_{0}$ of this third stage indicating the elasto-capillary stage followed by filament elongation is given in Table 2 for different PAM concentrations and flow rates $Q_{c}$ of silicone oil. It can be seen that variation of $Q_{c}$ has little effect on the onset of the third stage. The obtained results are in agreement with experimental observations of the evolution of the neck diameter of non-Newtonian aqueous solutions of polyethylene oxide in olive oil co-flowing in the microfluidic flow-focusing device [XueChen2020]. Moreover, the second stage of neck formation is well fitted by the scaling function $D_{f} \sim\left(t_{0}-t\right)^{\alpha}$ indicating the self-similar character of pinch-off process of the terminal droplet which was first discovered for Newtonian liquids [14, 23, J. Eggers, M. A. Herrada, and J. H. Snoeijer, J. Fluid Mech., 887, A19 (2020)]. In this case the scaling exponent ranges from $\frac{2}{3}$ for inviscid fluids to 1 when viscosity is dominant [2015Castrejon-Pita]. For viscoelastic fluids, the pinch-off process is suspended at the moment $t_{0}$ due to the balance between the elastic and capillary forces which in turn corresponds to beginning of stretching of the filament. The measured scaling exponents $\alpha$ are presented in Table 2 for different polymer concentrations. The higher the concentration, the lower $\alpha$, which is consistent with the data reported in refs. [du2017, XueChen2020] for flow focusing 
microsystems. In the former case, it was found that $0.2 \leq \alpha \leq 0.3$ for relative polymer concentration in the range $10 \geq c / c^{*} \geq 0.1$ [XueChen2020]. In our case, the scaling exponent $\alpha$ is in the range $0.28 \leq \alpha \leq 0.34$ for higher polymer concentrations, i.e. $60 \geq c / c^{*} \geq 10$ (see Table 2) which approaches the former upper limit .

Table 2. The onset $\left(t_{0}\right)$ of filament formation for different PAM concentrations and continuous phase flow rates, $Q_{d}=0.005 \mathrm{~mL} / \mathrm{min}$

\begin{tabular}{cccc}
\hline \hline \multirow{2}{*}{$\begin{array}{c}\text { PAM concentration, } \\
\text { wt. } \%\end{array}$} & \multicolumn{2}{c}{$t_{0}, \mathrm{~s}$} & Scaling exponent \\
\cline { 2 - 3 } & $\left(Q_{c}=0.1 \mathrm{~mL} / \mathrm{min}\right)$ & $\left(Q_{c}=0.1 \mathrm{~mL} / \mathrm{min}\right)$ & $\alpha$ \\
\hline 0.1 & $0.22 \pm 0.01$ & $0.24 \pm 0.01$ & $0.34 \pm 0.02$ \\
0.3 & $0.30 \pm 0.01$ & $0.29 \pm 0.01$ & $0.31 \pm 0.02$ \\
0.6 & $0.49 \pm \cdots$ & - & $0.28 \pm 0.02$ \\
\hline \hline
\end{tabular}

The observed difference of $\alpha$ may come from a stronger elastic response in a vicinity of the detachment point of the terminal droplet for more concentrated polymer solutions. Also, the difference in the obtained data may be coming from the difference of flow patterns in the flow focusing devices and coaxial capillaries. These claims need a more detailed study. A decrease in the scaling exponent for more concentrated polymer solutions correlates with an increase in their viscosity. At the same time, the shear thinning effect of the PAM solutions considered does not occur. The same conclusion was drawn for other non-Newtonian polymer solutions in the flowfocusing device [XueChen2020]. It can be explained by the axisymmetric flow of a thin part of the filament where shear rates are extremely small due to plug profile in the jet [J. Eggers and T. F. Dupont, J. Fluid Mech. 262, 205221 (1994)].

The elongated filament of the PAM aqueous solution appears when the drop begins to be pulled by the continuous phase at a fixed velocity $v_{d}$. This is indicated by the linear time dependences of the filament lengths $\left(L_{f}\right)$ for different polymer concentrations and silicone oil flow rates represented in Fig. 9. The filament elongation rates are determined by the slope of the linear interpolation of this curve. It can be seen that changes in PAM concentration in the range from 0.1 to $0.6 \mathrm{wt} . \%$ have almost no effect on the value of $v_{d}$, whereas it depends significantly on the flow rate of the continuous phase, as for instance, for $Q_{c}=0.1 \mathrm{~mL} / \mathrm{min}$, i.e. $v_{d}=2.5$ $\mathrm{mm} / \mathrm{s}$, and for $Q_{c}=0.2 \mathrm{~mL} / \mathrm{min}$, i.e. $v_{d}=5.396 \mathrm{~mm} / \mathrm{s}$. The observed values are clearly below the respective maximum velocities of the continuous phase, equal to 3.777 and $7.555 \mathrm{~mm} / \mathrm{s}$ which are the velocities in the capillaries before the two phases come in contact. This result is consistent with the results obtained [sessoms2009, sajeesh2014] for sufficiently large droplets. Besides, the elastic resistance of the filaments [24] as well as shear rate dependent viscosity of 
the polymer solution could modify velocity distribution the non-Newtonian droplet. In addition, the velocity has an influence on the size of the droplets formation: an increase in the flow rate of the continuous phase reduces the diameter of the droplets. The last points on the time dependence of $L_{f}(t)$ (see Fig. 9) correspond to the onset of filament instability. This indicates that an increase in polymer concentration and continuous phase flow rate favors the growth of filament length.

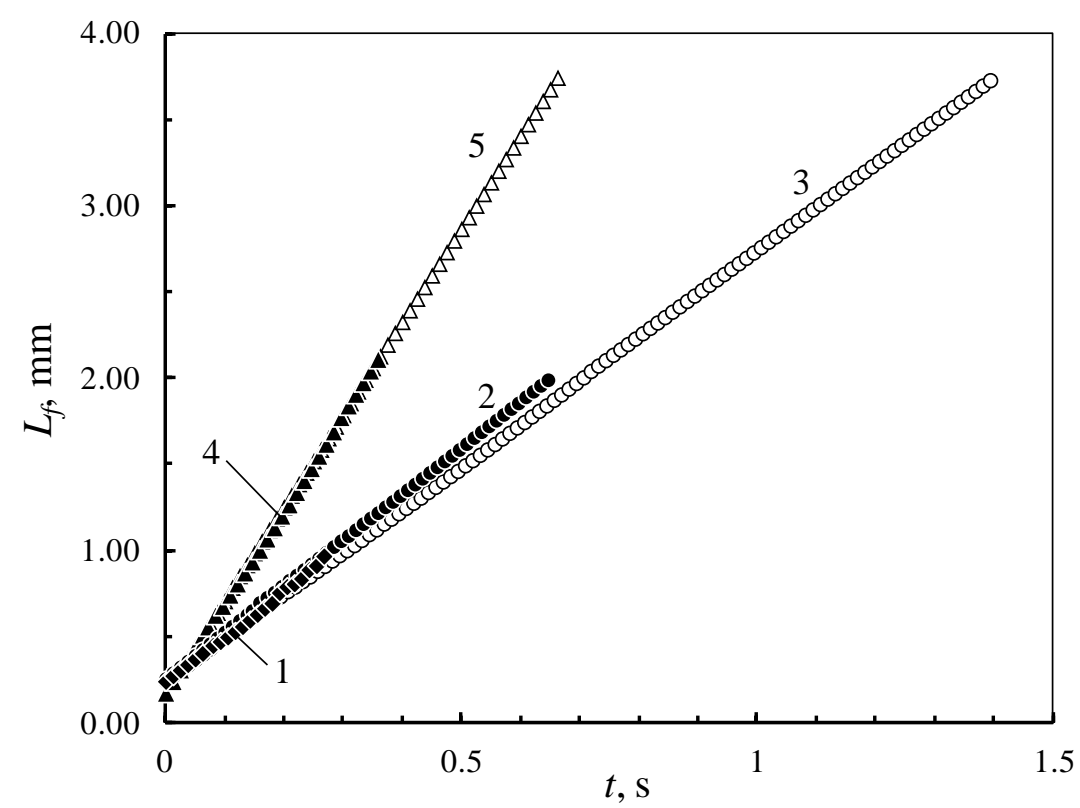

Figure 9. Variation of the filament length with terminal droplet for different PAM concentration and silicone oil flow rate combinations: 0.1 (line 1, open circles), 0.3 (2, filled circles) wt.\% for $\mathrm{Q}_{\mathrm{c}}=0: 1 \mathrm{~mL}$ and 0.1 (3, open triangles), $0.3 \mathrm{wt} . \%$ (4, filled triangles) for $\mathrm{Q}_{\mathrm{c}}=0: 2 \mathrm{~mL} / \mathrm{min}$.

According to Fig. 9, the length of the polymer filament can be represented as a linear function of time

$L_{f}(t)=L_{f 0}+v_{d}\left(t-t_{0}\right)$,

where $L_{f 0}$ is the initial filament length at the onset time $t_{0}$ (see Table 2). The initial filament length $L_{f 0}$ at time $t_{0}$ was approximately $200 \mu \mathrm{m}$ in all cases. If the flow of dispersed fluid going from filament towards the terminal droplet is negligible, the current filament diameter $D_{f}(t)$ can be estimated from the incompressibility condition. For the PAM aqueous solutions under consideration, this condition is roughly justified by the fact that Poisson ratio of PAM gels is about 0.48 [T. Boudou, J. Ohayon, C. Picart and P. Tracqui, An extended relationship for the characterization of Young's modulus and Poisson's ratio of tunable polyacrylamide gels, Biorheology 43 (2006), 721-728]. Taking into account that $L_{f}(t) D_{f}^{2}(t)=L_{f 0} D_{f 0}^{2}$ we obtain the following relationship:

$D_{f}(t)=D_{f 0}\left[1+\frac{v_{d}}{L_{f 0}}\left(t-t_{0}\right)\right]^{-1 / 2}$ 
where $D_{f 0}$ are the filament diameter at the initial time $t_{0}$.

On the other hand, the decrease in the filament diameter can be caused by the overflow of the polymer solution into the terminal droplet due to the capillary pressure $\sigma_{r}=-2 \gamma / D_{f}(t)$ in the filament ( $\gamma$ is the interfacial tension). This effect was studied in detail on the example of the self-thinning bridges of polymer solution between the beads on the stretched liquid thread [22, $23,39]$. In this case, the decrease of filament diameter is described by the following exponential dependence [22]:

$D_{f}(t)=D_{f 0} \exp \left(-\frac{t-t_{0}}{3 \theta}\right)$

where $\theta$ is the stress relaxation time specified in the framework of Chilcott-Rallison version of the Oldroyd model [40]. The equation (3), however, does not take into account the effect of the reduction of the filament diameter caused by its stretching due to the motion of the terminal droplet under the action of the continuous phase (Eq. (2)).
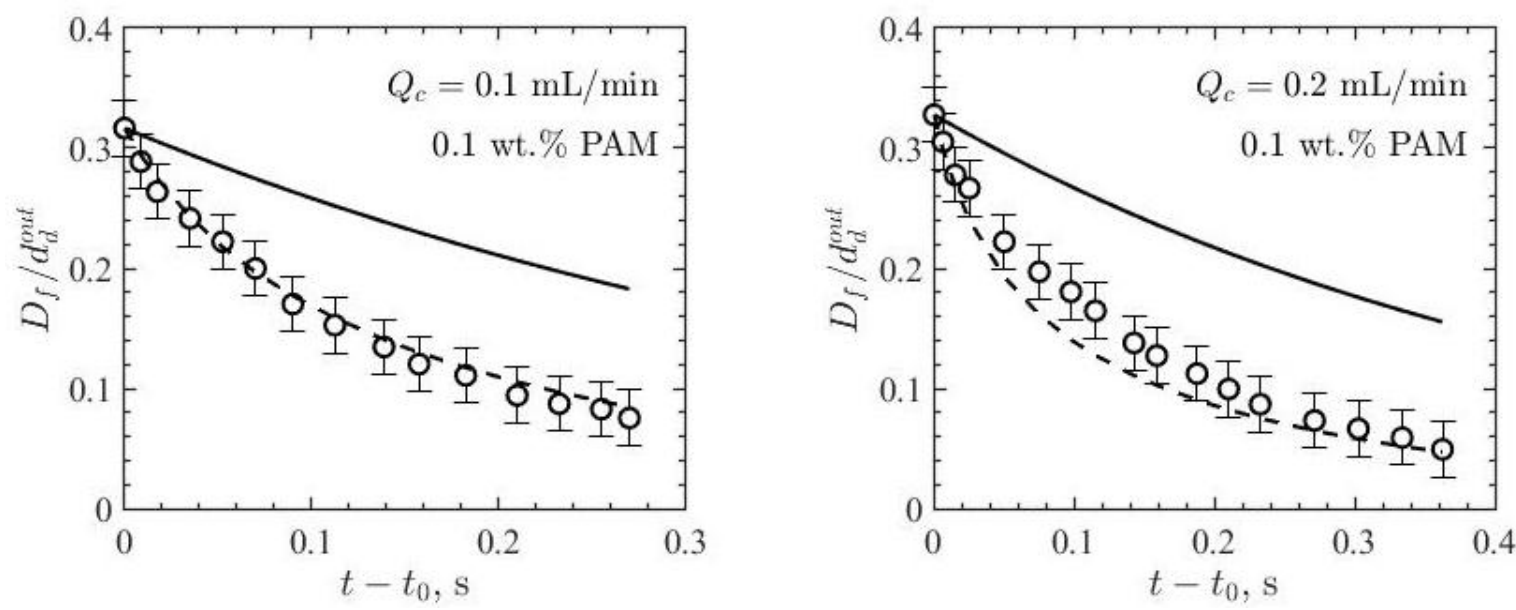

(a)
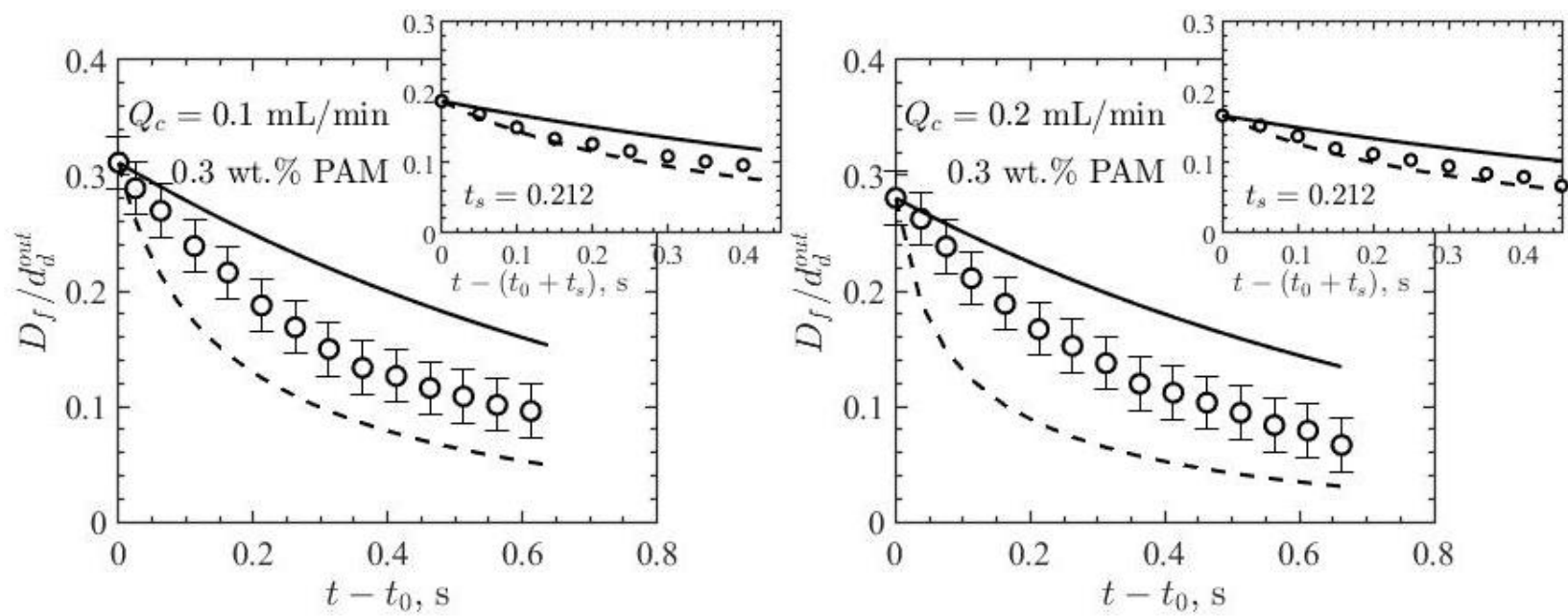

(b)

Fig. 10. Evolution of the dimensionless diameter $D_{f} / d_{d}^{\text {out }}$ (circle symbols) for the polymer solution with PAM concentration of $0.1 \mathrm{wt} . \%$ (a) and $0.3 \mathrm{wt} . \%$ (b) at silicone oil flow rates $Q_{c}=$ 0.1 (left) and $0.2 \mathrm{~mL} / \mathrm{min}$ (right). The solid and dashed lines correspond to Eq. (3) and Eq. (6), 
respectively. The inset plots were plotted by using parameters of only the late time $\left(t_{\mathrm{s}}=0.212 \mathrm{~s}\right)$ fit.

The combined effect can be estimated from the rate of change in the filament volume

$\frac{d}{d t}\left(L_{f} D_{f}^{2}\right)=-\frac{2}{3 \theta} L_{f} D_{f}^{2}$.

This equation claims that rate of change in filament volume is in balance with the rate of leakage of polymer solution into the terminal droplet at a current filament length: $\frac{d}{d t} V_{\text {leak }}=2 L_{f} D_{f} \frac{d D_{f}}{d t}$, where $\frac{d D_{f}}{d t}=-\frac{D_{f}}{3 \theta}$ according to Eq. (3). Equation (4) can be rearranged as following $\frac{d}{d t} \ln \left(D_{f} L_{f}^{1 / 2}\right)=-\frac{1}{3 \theta}$.

The integration of last equation gives the following result:

$D_{f}(t)=-D_{f 0}\left[1+\frac{v_{d}}{L_{f 0}}\left(t-t_{0}\right)\right]^{-1 / 2} \exp \left(-\frac{t-t_{0}}{3 \theta}\right)$

where Eq. (1) was taken into account. Eq. (6) looks alike eq. (3) but in Eq. (6) the preexponential factor $D_{f 0}$ corresponding to the filament diameter in the initial moment $t_{0}$ was replaced by the time-dependent value $D_{f}(t)$ given by Eq. (2).

Equations (3) and (6) allow us to estimate which of the mentioned mechanisms of decrease of the filament diameter in the dripping mode - either a pure squeezing-out of polymer solution into the terminal droplet under the capillary compression or the combined effect involving filament stretching - is closer to the experimental data. Fig. 10 shows experimental and estimated dependences of the filament diameter on time in the dripping mode for two aqueous PAM solutions with polymer concentrations of 0.1 and 0.3 wt. \% and for silicone oil flow rates $Q_{c}=0.1$ and $0.2 \mathrm{~mL} / \mathrm{min}$. In these plots, the circle symbols represent experimental data while solid and dashed lines correspond to Eqs. (3) and (6), respectively. The mean values of Table II of Section $\mathrm{C}$ were used as relaxation times $\theta$, which were measured for the polymer solutions considered, under the condition of steady-state jet flow.

The best agreement with the combined mechanism of the filament diameter evolution described by Eq. (6) is achieved for solutions with the lowest polymer concentration i.e. with a volume fraction of $0.1 \mathrm{wt} . \%$. In this case, factors associated with both jet stretching and capillary compression contribute to the decrease of the filament diameter. On the other hand, at a higher PAM concentration of $0.3 \mathrm{wt} \%$, the influence of filament stretching was reduced. To explain these observations, we have to remember that the lowest polymer concentration corresponds to a semi-diluted solution with the smallest concentration ratio $c / c^{*} \cong 10$ whereas at $c=0.3 \mathrm{wt} . \%$ this ratio is noticeably higher, $c / c^{*} \cong 30$ (see Section II). 
The effect of the exponential decrease of the filament diameter due to the outflow of the dispersed phase into the terminal droplet has been firmly proven just for dilute polymer solutions under conditions a fixed-length jet $[12,14,23,25]$ as well as in the stretching mode under the action of gravitational forces [C. Clasen, J. Bico, V.M. Entov, G.H. Mckinley 'Gobbling drops': the jetting-dripping transition in flows of polymer solutions, J. Fluid Mech., 636, 5-40 (2009)]. At the same time, to the best of our knowledge, the decrease in the filament diameter due to the motion of the terminal droplet under the action of the incoming continuous phase in a channel has not been taken into account so far. Therefore, the good agreement of Eq. (6) with the experimental data shown in Fig. 10a indicates the validity of this simple representation for the smallest polymer concentration considered. The failure of such a correspondence for a more concentrated polymer solution suggests that the proposed equation does not work at least for an insufficiently thin filament. In this case, the rheological response of the squeezed polymer solution requires more time for stretching the temporal network of the entangled polymer chains. This happens when the elastic stress in a filament exceeds capillary and viscous forces [Bhattacharjee, P. K., Nguyen, D. A., McKinley, G. H. \& Sridhar, T. Extensional stress growth and stress relaxation in entangled polymer solutions. J. Rheol. 47, 269-290 (2003)]. Apparently, this condition was not met for a thick and insufficiently long section of the filament, which is close to the inner capillary. Therefore, the time $t_{0}$ corresponding to the transition to the third stage of evolution of the filament diameter (see Figs. 6, 7 and Table II), does not correspond to the beginning of the flow of the semi-dilute polymer solution. As a result, Eq. (6) is not consistent with experiment for PAM concentration of $0.3 \mathrm{wt}$.\%. However, if the onset of the filament diameter evolution is shifted to a later time $t_{0}+t_{s}$, at which the filament is sufficiently long and thin, we could expect that the elongational flow caused by the capillary compression will rise with the filament stretching, and its diameter $D_{f}(t)$ should follow Eq. (6). This assumption was confirmed by the plots presented in the insets of Fig. 10b, where the reference time has been shifted by $t_{s}=0.212 \mathrm{~s}$. In this case, the initial ratios $L_{f}\left(t_{0}+t_{s}\right) / D_{f}\left(t_{0}+t_{s}\right)$ of the filament length to its diameter corresponds to 11.46 and 22.42 at $Q_{c}=0.1$ and $0.2 \mathrm{~mL} / \mathrm{min}$, respectively. The obtained results indicate a qualitative difference in the evolution of the filament diameter formed between the tip of the inner capillary and the terminal droplet due to different viscoelastic response of the considered semi-dilute polymer solutions of different concentrations.

\section{Jetting}

1. Capillary instability 
The diagram of flow modes of the PAM aqueous solution presented in Fig. 6 indicates that an increase in polymer concentration and/or the relative flow rate of the continuous medium extends the jetting flow mode area. On the contrary, the smaller the concentration of polymer the solution, the larger flow rate of the continuous phase is required to form a stable jet. The images presented in Fig. 6 depict just a small part of the jet close to the inner capillary outlet. The stepwise shift of the microdevice relative to the microscope field made it possible to record the subsequent variations in the jet structure along the capillary axis. Figures $5 \mathrm{c}$ and 11 show sequences of typical snapshots for each microscope position, showing different stages of jet evolution for 0.1 and 0.6 wt.\% PAM aqueous solutions at continuous phase flow rate of $Q_{c}=0.8$ and $0.6 \mathrm{~mL} / \mathrm{min}$, respectively. Each subsequent position corresponds to a shift of the microdevice by $1.8 \mathrm{~mm}$ corresponding to the microscope field of view. By the example of Fig. 6 , it can be seen that for the first few millimeters (see positions 1-3) the jet remains stable and does not change its shape over time. However, at a distance $5.5 \mathrm{~mm}$ away from the source, capillary waves become visible on the jet interface. Some of the most unstable wave trains are then transformed into a set of beads connected by thin filaments (positions 4-8). As one moves away from the inner capillary tip, the transverse size of the beads $D_{b}$ gradually stabilizes while $D_{f}$ the cross-sectional dimensions of the filaments between the beads continues to decrease (positions 8-10). This jet structure differs from those formed by the rapid separation of solid plates wetted with the same polymer solution in a quiescent fluid [12, 20, 21, 23, 26] or by its stretching in a four-roll mill [35]. In the case considered in Fig. 11, the jet length exceeds $30 \mathrm{~mm}$ before it decays. However, its length decreases with decreasing polymer concentration as it is shown in Fig. 5c.
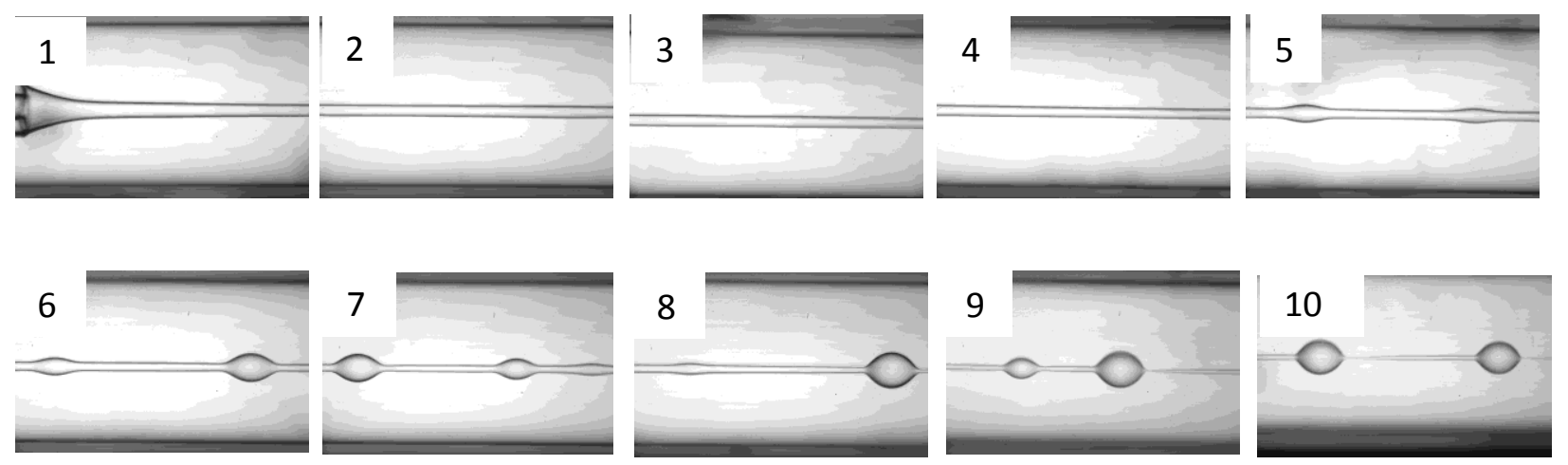

Figure 11. Evolution of the jet structure of $0.6 \mathrm{wt} . \%$ PAM aqueous solution for a silicone oil flow rate $Q_{c}=0.6 \mathrm{~mL} / \mathrm{min}$. Images 1 to 10 were taken $1.8 \mathrm{~mm}$ from the previous one further down in the flow.

The main difficulty in studying the dynamic behavior of the polymer jet co-flowing along with the external viscous medium is the changes of the jet structure due to the continuous formation of novel beads. The linear analysis of capillary instabilities of viscoelastic jets 
provides an adequate understanding of the period of the fastest growing modes of the jet perturbations [J. Eggers and T. F. Dupont, J. Fluid Mech. 262, 205221 (1994)]. Due to the growth of elastic stress in the jet to a level that is comparable with capillary pressure and viscoelastic stress, such perturbations are quickly transformed into spherical beads [10, 14]. At the beginning, the distance between the beads is consistent with the periodicity of the fastest growing modes. But then the liquid beads start to migrate along the jet and merge [10; A.M. Ardekani, V. Sharma, and G.H. Mckinley, J. Fluid Mech. 665, 46-56 (2010)]. This complicates the measurement of mean filament lengths between them and makes statistical analysis more appropriate than linear stability analysis.

The evolution of the jet structure could be monitored at fixed positions along the capillary axis in the reference frame. On the other hand, it may be reasonable to track the jet transverse dimensions as a function of time. Figure 12 shows the jet transversal fluctuations for $0.6 \mathrm{wt} . \%$ PAM solution at distances $x=3.6,12.6$, and $21.6 \mathrm{~mm}$ from the inner capillary tip for a silicone oil flow rate of $Q_{c}=0.6 \mathrm{~mL} / \mathrm{min}$. The equivalent jet diameter $D^{*}$ (see eq. (7)) has been used as a scaling length.

The virtually invariability of the jet diameter at the point $x=3.6 \mathrm{~mm}$ (see the black curve in Fig. 12) agrees with the images 1-4 shown in Fig. 11. At a larger distance $x=12.6 \mathrm{~mm}$ there are random oscillations of the jet diameter (red curve), caused by the capillary waves of different amplitudes and wavelengths. Even farther $(x=21.6 \mathrm{~mm})$, different high pulses corresponding to different beads sizes are observed (blue curve). The beads are several times thicker than the connecting filaments (the lower level of the blue curve). Figure 12 shows also that the filament's diameter decreases with its distance from the inner capillary outlet.

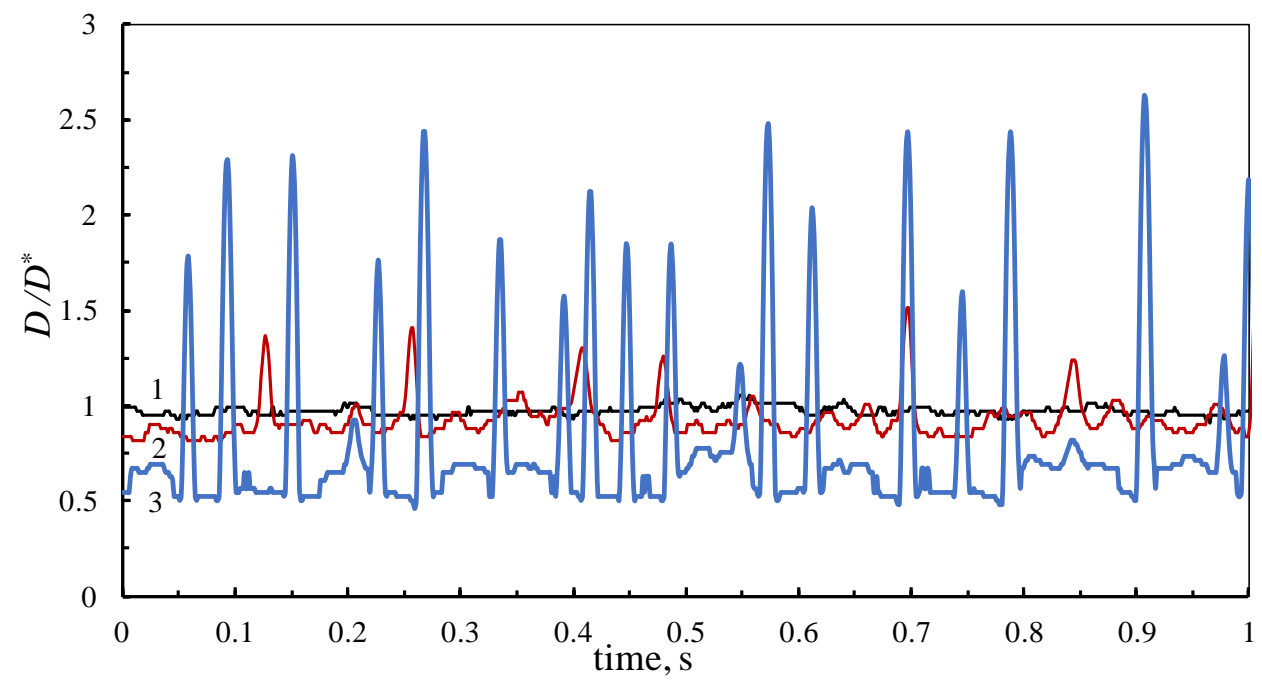

Figure 12. Fluctuations of the dimensionless lateral jet size (filaments and beads) of $0.6 \mathrm{wt} . \%$ aqueous PAM solution at different points $x$ along the capillary axis: $x=3.6$ (black curve 1), 12.6 (brown 2), and $21.6 \mathrm{~mm}$ (blue 3) at the silicone oil flow rate $Q_{c}=0.6 \mathrm{~mL} / \mathrm{min}$. 
Figure 13 shows the typical evolution of the mean diameter of filaments as a function of the distance from the inner capillary tip for the $0.6 \mathrm{wt} \%$ PAM aqueous solution and for a silicone oil flow rate of $Q_{c}=0.6 \mathrm{~mL} / \mathrm{min}$. The arrows indicate points $x=3.6,12.6$, and $21.6 \mathrm{~mm}$ considered in Fig. 12. The evolution sequence of the filament diameter $D_{f}$ can be subdivided into three steps.

In the first step, the speed of jet propagation increases from the mean velocity in the inner capillary to a certain steady value. At the threshold between stages I and II (left vertical line in Fig. 13), the equivalent jet diameter $D^{*}$ is considerably smaller than the inner diameter of the capillary $d_{c}$. This allows us to assume that the jet steady-state velocity is close to the maximum velocity of the continuous phase, $v_{c}^{\max }$. The $D^{*}$ value can be estimated from the conservation of the flow rate of the polymer solution $Q_{d}=v_{c}^{\max } S^{*}$, where $S^{*}=\frac{1}{4} \pi D^{* 2}$ is the cross-section area of the corresponding part of the jet. On the other hand, the maximum Poiseuille flow velocity of the continuous phase (which is a Newtonian fluid flowing in a cylinder) in the external capillary is equal to $v_{c}^{\max }=2 U_{c}=2 \frac{Q_{c}}{S_{c}}$, where $U_{c}$ is the mean velocity, and $S_{c}=\frac{1}{4} \pi d_{c}^{2}$ it the capillary cross-section area. Hence, we find:

$D^{*}=d_{c}\left(\frac{Q_{d}}{2 Q_{c}}\right)^{1 / 2}$.

In particular, for flow rates of the polymer solution $Q_{d}=0.005 \mathrm{~mL} / \mathrm{min}$ and silicon oil of $Q_{c}=$ 0.6, 0.8 and $1.0 \mathrm{~mL} / \mathrm{min}$ the jet equivalent diameter $D^{*}$ should be equal to $64.6,55.9$, and 50.0 $\mu \mathrm{m}$, respectively. The image analysis confirms that such lateral dimensions of the jet were indeed present between the first and second step of the jet propagation regardless of the polymer concentration (see the black curve in Fig. 12).

The transition from the second to the third step of jet propagation is determined by the first appearance of noticeable fluctuations of its interface with the silicon oil (see curve 2 in Fig. 12). During this step, beads on the string begin to appear, while the diameter of the connecting filaments reduces exponentially due to flow of the polymer solution into the growing beads (see the insets in Fig. 13) [22-24]. 


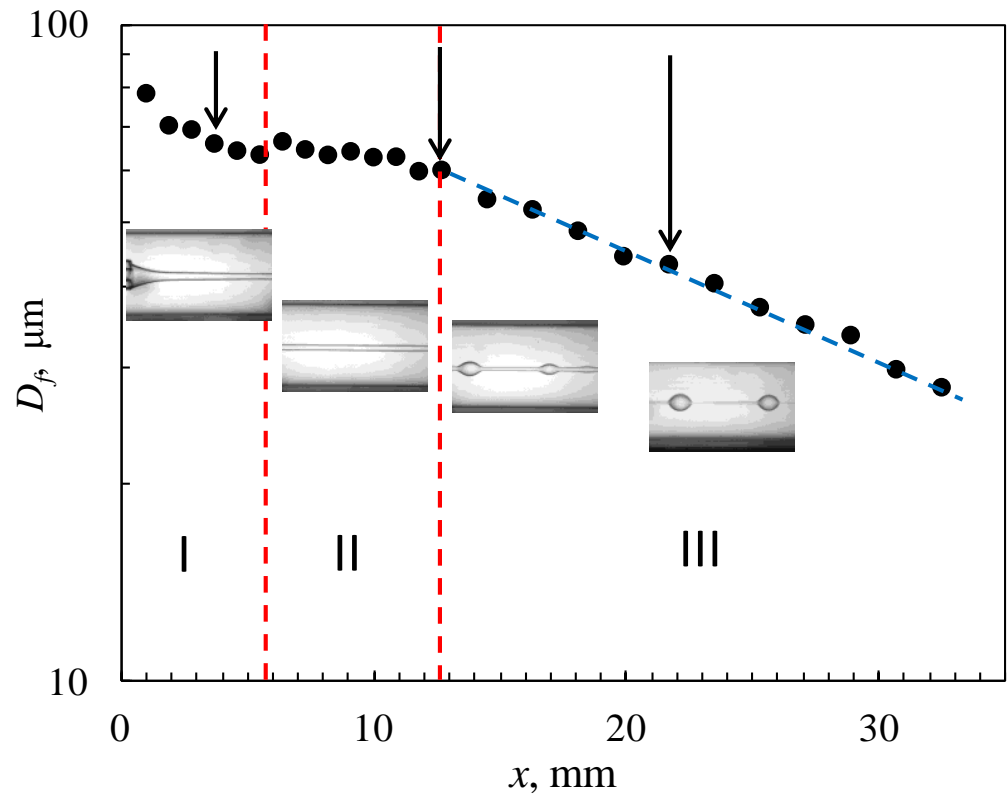

Figure 13. Diameter jet filaments of $0.6 \mathrm{wt} . \%$ PAM aqueous solution for a silicone oil flow rate of $0.6 \mathrm{~mL} / \mathrm{min}$ versus distance $x$ from the inner capillary top. Red dashed lines separate different stages of jet propagation. The insets indicate the corresponding jet morphology.

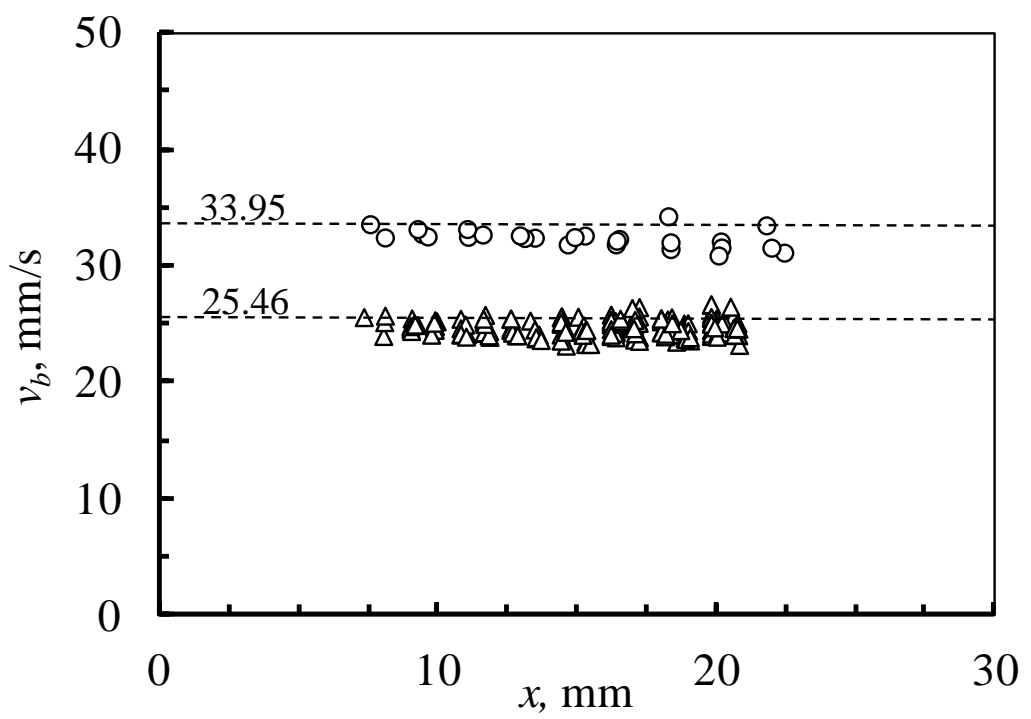

Figure 14. Beads velocity for $0.3 \mathrm{wt} \%$ PAM aqueous solution jet and silicone oil flow rates $Q_{c}$ $=0.6$ and $0.8 \mathrm{~mL} / \mathrm{min}$ represented by triangle and circles respectively. The dotted lines indicate the corresponding maximum Poiseuille flow velocity of the silicone oil.

Figure 14 shows the measured bead velocities along the capillary for $0.3 \mathrm{wt} \%$ PAM aqueous solution at two flow rates of silicone oil of $Q_{c}=0.6$ and $0.8 \mathrm{~mL} / \mathrm{min}$. Their values are close to the maximum velocity of the continuous medium in the external capillary defined as $v_{c}^{\max }=\frac{8 Q_{c}}{\pi d_{c}^{2}}$. Indeed, for flow rates $Q_{c}=0.6$ and $=0.8 \mathrm{~mL} / \mathrm{min}$ the calculated maximum velocity should be equal to $v_{c}^{\max }=25.46$ and $33.95 \mathrm{~mm} / \mathrm{s}$, respectively, which is in agreement with the presented experimental data. This indicates also the steady-state character of jet propagation 
during the third step of the process. This consistency between the experimental and estimated jet velocities has been observed for all PAM concentrations and silicone oil flow rates considered.

Due to the steady-state jet motion, the decrease in filament diameter is caused by the flow of polymer solution into the emerging beads under the capillary compression $\sigma_{r}=-2 \gamma / D_{f}$, as in the case of the quiescent viscous thread. For this reason, the change in filament diameter $D_{f}(t)$ also obeys the exponential decay (see Fig. 13) [22, 23, 38].
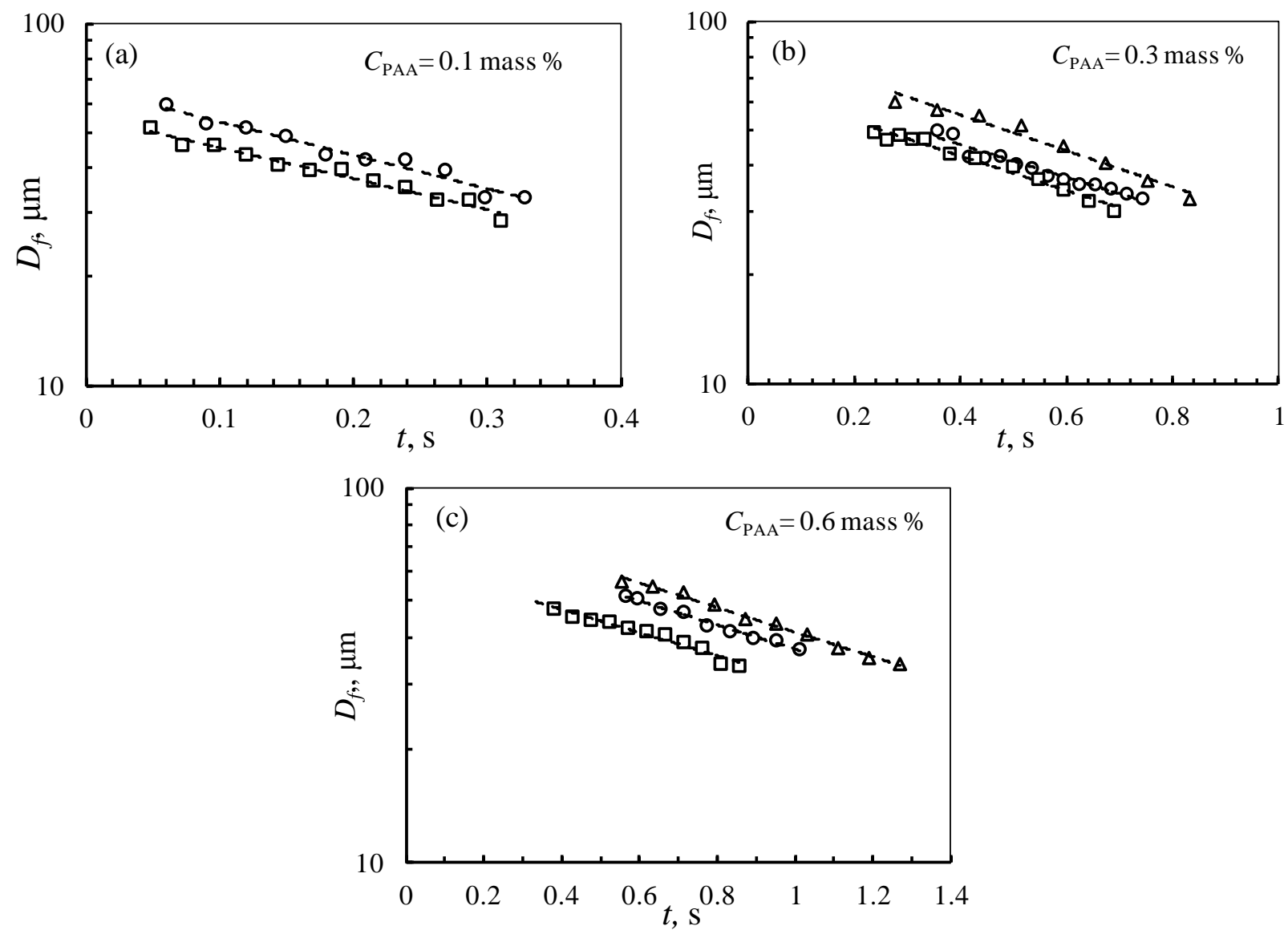

Figure 15. Time dependence of filament diameter at the third stage of jet propagation for PAM aqueous solutions with polymer fractions of 0.1 (a), 0.3 (b) and $0.6 \mathrm{wt} . \%$ (c) and silicone oil flow rates $Q_{c}=0.6$ (triangle), 0.8 (circles) and $1.0 \mathrm{~mL} / \mathrm{min}$ (squares). The red dashed lines correspond to the relationship $D_{f} \sim \exp \left(-\frac{t-t_{0}}{3 \theta}\right)$.

Figure 15 shows thinning of the filament diameter between the beads with time $t=$ $x / v_{c}^{\max }$ during the third step for three PAM concentrations of $0.1,0.3$, and $0.6 \mathrm{wt} . \%$ and for silicone oil flow rates of $Q_{c}=0.6,0.8$, and $1.0 \mathrm{~mL} / \mathrm{min}$. The linear dependence in the semilogarithmic coordinates indicates the exponential behavior of $D_{f}(t)$ function. The polymer solutions relaxation times $\theta$ were estimated from Eq. (3). The obtained data are presented in Table 3. As can be seen, the relaxation times are almost independent of the flow rate $Q_{c}$ of the continuous phase. This is not surprising since the jet motion is in a steady-state in all cases. Moreover, the average relaxation time $\theta$ increases significantly with increasing polymer concentration. 
Table 3. Relaxation times of jets produced with different PAM aqueous solutions.

\begin{tabular}{ccccc}
\hline \hline \multirow{2}{*}{$\begin{array}{c}\text { PAM fraction, } \\
\text { wt.\% }\end{array}$} & \multicolumn{3}{c}{ Relaxation times, $\mathrm{c}$} & $\begin{array}{c}\text { Average relaxation } \\
\text { time }\end{array}$ \\
\cline { 2 - 5 } & $Q_{\mathrm{c}=0.6 \mathrm{~mL} / \mathrm{min}}$ & $Q_{\mathrm{c}}=0.8 \mathrm{~mL} / \mathrm{min}$ & $Q_{\mathrm{c}}=1.0 \mathrm{~mL} / \mathrm{min}$ & $\theta, \mathrm{s}$ \\
\hline $\mathbf{0 . 1}$ & - & 0.156 & 0.169 & $0.162 \pm 0.006$ \\
$\mathbf{0 . 3}$ & 0.291 & 0.305 & 0.320 & $0.305 \pm 0.014$ \\
$\mathbf{0 . 6}$ & 0.439 & 0.449 & 0.469 & $0.452 \pm 0.016$ \\
\hline \hline
\end{tabular}

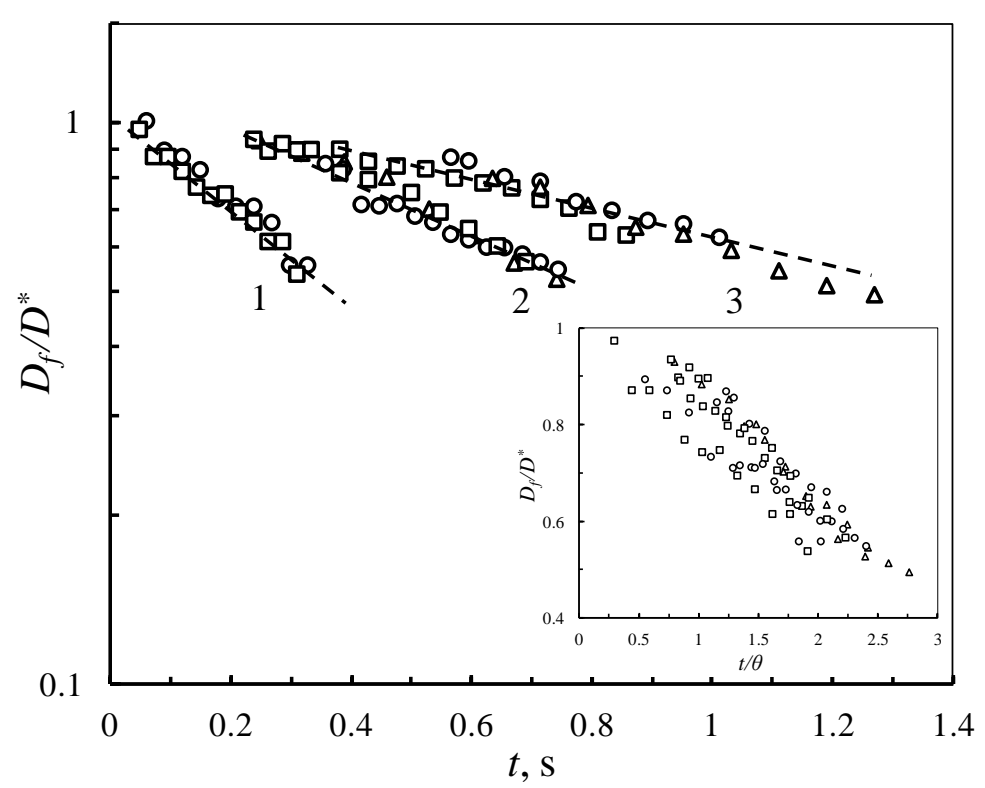

Figure 16. The time dependence of the reduced filament diameters for the aqueous PAM solutions with polymer concentration of 0.1 (1), 0.3 (2), and 0.6 wt.\% (3) and different silicone oil flow rates: $Q_{c}=0.6$ (triangles), 0.8 (circles), and $1.0 \mathrm{~mL} / \mathrm{min}$ (squares). The red dashed lines correspond to the relationship $D_{f} / D * \sim \exp \left(-\frac{t-t_{0}}{3 \theta}\right)$. The inset shows dependence of $D_{f} / D^{*}$ with the dimensionless time $t / \theta$.

It is worth noting that the dependences shown in Fig. 15 can be reduced to single lines if the filament diameter $D_{f}$ is made dimensionless by dividing by the equivalent diameter $D^{*}$ calculated from Eq. (7). The corresponding dependences are presented in Fig. 16 for the three polymer solutions considered with PAM concentration of $0.1,0.3$, and 0.6 wt. \%. It can be seen that the dimensionless filament diameter $\bar{D}_{f}=D_{f}(t) / D^{*}$ ceases to depend on the continuous phase flow rate. In this representation, the slope of $\bar{D}_{f}(t)$ depends on the polymer concentration only, which indicates indirectly the steady-state character of the jet flow. The above data can be collected into a single master curve in the coordinates $D_{f} / D^{*}$ vs. $\tau=t / \theta$ by using the 
dimensionless time $\tau=t / \theta$ (see the inset in Fig. 16). Some scatter of the experimental data is due to the difference in the instability moments of jets with different polymer concentrations.

\section{Beads formation}

Fig. 17 shows the measured evolution of the lateral dimensions of beads and the filaments linking them for PAM aqueous solutions with different polymer concentrations $(0.3$ and 0.6 $\mathrm{wt} \%$ ) and for constant silicone oil flow rate $Q_{\mathrm{c}}=1.0 \mathrm{~mL} / \mathrm{min}$ (recall that the flow rate of polymer solution coming from the inner capillary is always constant and equal to $Q_{d}=0.005 \mathrm{~mL} / \mathrm{min}$ ). The open symbols correspond to the average filament diameter $D=D_{f}$, whereas the filled symbols correspond to the beads maximum lateral size $D=D_{b}$. Each subsequent curve of the filled symbols corresponds to the beads which were born on the filaments at later time points. At the beginning, the beads size increases rapidly but then their growth rate slows down. This process is accompanied by thinning of the filaments linking successive beads, which eventually leads to the jet disintegration. The comparison of Figs. 17a and $17 \mathrm{~b}$ reveals that an increase in polymer concentration leads to a later time appearance of the first beads which indicates a stronger stability of the corresponding jet.
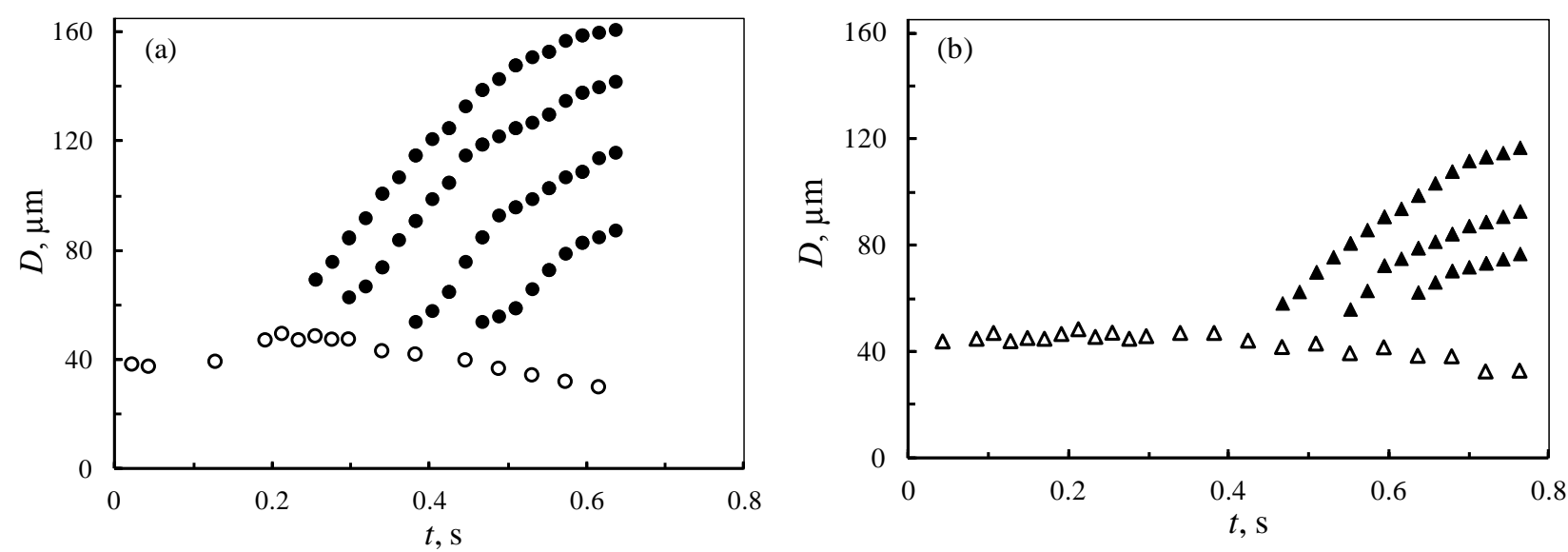

Figure 17. Evolution of the lateral dimensions of beads (filled symbols) and thin filaments (open symbols) during flow of polymer solution with PAM concentrations of 0.3 (a) and 0.6 (b) wt.\% and for a silicone oil flow rate of $Q_{c}=1.0 \mathrm{~mL} / \mathrm{min}$.

The birth of new beads between previously formed ones leads naturally to a shortening of the filament length $L_{f}$. This is confirmed by the data shown in Fig. 18 for an aqueous PAM solution with polymer concentration of $0.3 \mathrm{wt} \%$ for three silicone oil flow rates $Q_{c}=0.6,0.8$ and $1.0 \mathrm{~mL} / \mathrm{min}$. The results are presented in the dimensionless form as dependences of the dimensionless filament length $\bar{L}_{f}=L_{f} / D^{*}$ on the dimensionless time $\tau_{0}=t_{0} / \theta$ of a bead birth $\left(D^{*}\right.$ is the equivalent jet diameter given by Eq. (7)). In the case under consideration, the average 
relaxation time is $\theta=0.305$ (see Table 3 ). The representation of this dependence in logarithmic coordinates (see the inset in Fig. 18) shows that $\bar{L}_{f}\left(\tau_{0}\right)$ corresponds to a power law function

$\bar{L}_{f}=\bar{L}_{0} \tau_{0}^{-\beta}$

where the index $\beta$ is almost independent of the flow rateflow rate $Q_{c}$ of the continuous phase and is equal to $\beta=1.5 \pm 0.11$. The dimensionless factor $\bar{L}_{0}$ in equation (8) corresponds roughly to 71.3 .

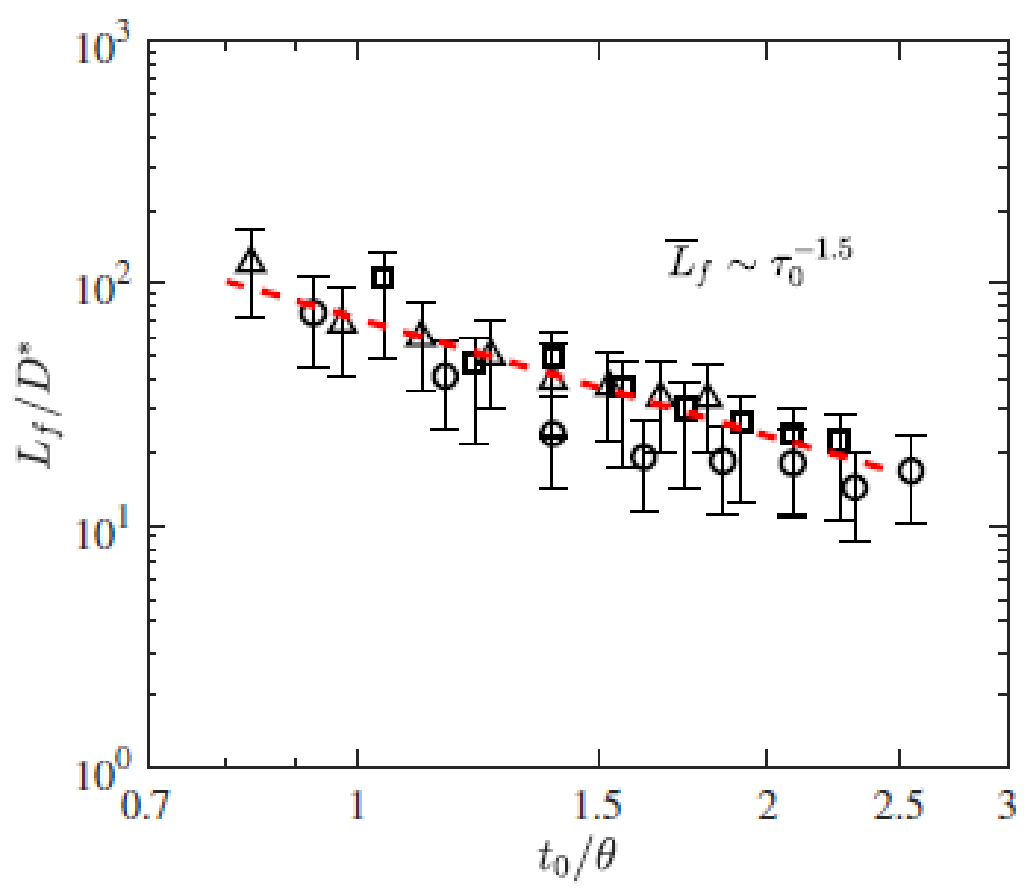

Figure 18. Dimensionless bead spacing (filament length) versus dimensionless bead birth time $t_{0} / \theta$ for $0.3 \mathrm{wt} \%$ PAM aqueous solution for three silicone oil flow rates: $Q_{c}=0.6$ (circle symbols), 0.8 (squares), and $1.0 \mathrm{~mL} / \mathrm{min}$ (triangles). The red dashed lines correspond to the relationship $\bar{L}_{f} \sim \tau_{0}^{-1.5}$.

In steady-state flow conditions, the thinning of the filament diameter, as was noted, is attributed to the flow of polymer solution into the neighboring beads under the capillary compression $\sigma_{r}=-2 \gamma / D_{f}$. This causes the dimensionless filament diameter $\bar{D}_{f}$ to decrease exponentially, as

$\bar{D}_{f}(\tau)=\exp \left(-\frac{1}{3}(\tau-\bar{T})\right)$

where $\bar{T}=T / \theta$ is the dimensionless time of the onset of instability development. By definition, at this point $\bar{D}_{f}(\bar{T})=1$.

It is natural to assume that change in the bead volume occurs only due to a thinning in the filament diameter caused by the capillary compression and does not depend on its length changes. As a result, for the symmetric jet fragment containing a spherical bead (see Fig. 3a) we have $D_{b}^{2} d D_{b}=-L_{f} D_{f} d D_{f}$. Taking into account Eqs. (8) and (9), we get the following equation: 
$\bar{D}_{b}^{2} \frac{d \bar{D}_{b}}{d \tau}=\frac{1}{3} \bar{L}_{0} \tau^{-\beta} \exp \left[-\frac{2}{3}(\tau-\bar{T})\right]$

where $D^{*}$ and $\theta$ are the length and time scales, respectively. As an initial condition, we considered that the lateral size of a bead at the initial time $\tau_{0}$ corresponds to filament diameter, $\bar{D}_{b}\left(\tau_{0}\right)=\exp \left[-\frac{1}{3}\left(\tau_{0}-\bar{T}\right)\right]$. Taking $\beta=1.5$, we obtain the solution of Eq. (10) as $\bar{D}_{b}(\tau)=\left\{C-2 \bar{L}_{0} \tau^{-1 / 2} \mathrm{e}^{\frac{2}{3} \bar{T}}\left[\mathrm{e}^{-\frac{2}{3} \tau}+\left(\frac{2}{3} \pi \tau\right)^{1 / 2} \operatorname{erf}\left(\left(\frac{2}{3} \tau\right)^{1 / 2}\right)\right]\right\}^{1 / 3}$

where the constant $C$ is determined from the initial condition. The evolution of the dimensionless size of beads born at different dimensionless times $\tau_{0}=0.9,1.1,1.3,1.5,1.7,1.9,2.1$ is shown in Fig. 19 (solid curves). The calculations were performed for $\bar{T}=0.7$ and $\bar{L}_{0}=71.3$. It can be seen that the theoretical curves given by Eq. (11) agree quite well with the experimental data for PAM aqueous solutions of different polymer concentrations (0.3 and $0.6 \mathrm{wt} . \%)$ and at different silicone oil flow rates from 0.6 to $1.0 \mathrm{~mL} / \mathrm{min}$. The lower dashed line corresponds to the exponential decrease of the filament diameter given by Eq. (9).

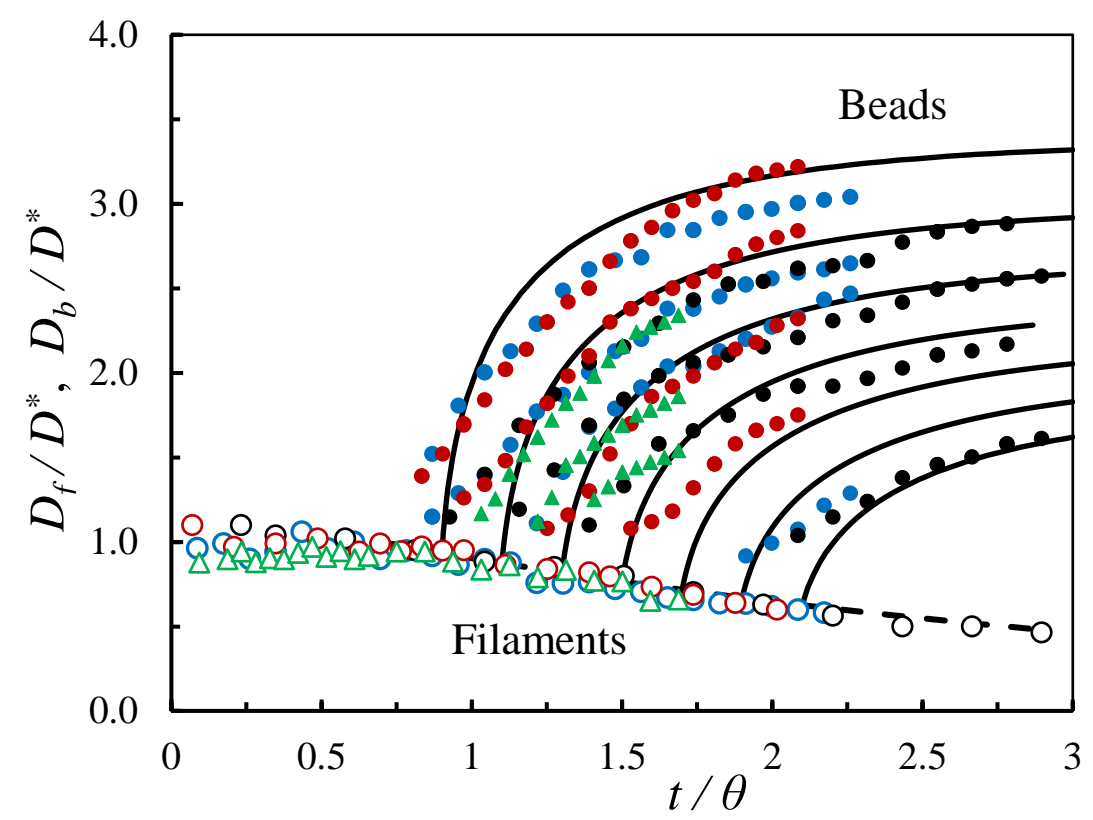

Figure 19. Experimental and theoretical time dependences of the lateral sizes of beads (filled symbols and solid lines, respectively) and filaments (open symbols and dashed lines) for polymer aqueous solution with PAM concentration of $0.3 \mathrm{wt} . \%$ at the silicone oil flow rates $Q_{c}=0.6$ (black circles), 0.8 (blue circles), and $1.0 \mathrm{~mL} / \mathrm{min}$ (red circles), and $0.6 \mathrm{wt} . \%$ at $Q_{c}=1.0$ (green triangles).

It was shown above (see the inset in Fig. 16) that the dimensionless representation of filament diameter and time, as $\bar{D}=D / D^{*}$ and $\tau=t / \theta$ collapses the experimental data on a single master curve, which does not depend on the concentration of the polymer solution and on the continuous medium flow rate. Fig. 19 generalizes this result to the evolution of the lateral 
bead sizes which emerged at different times. It is seen that, in this representation, the relative bead size $\bar{D}_{b}$, does not depend either on the continuous medium flow rate or polymer concentration: the obtained experimental data are virtually consistent, and their time dependence is quite close to the calculated curves for a given instant $\tau_{0}$ of bead birth. The average relaxation times for different solutions were taken from Table 3.

The obtained results indicate the universal character of evolution of filament and bead lateral sizes during capillary instability of jet of polymer solution co-flowing with Newtonian continuum medium in the coaxial capillary microsystem.

\section{Conclusions}

the concentrations of PAM 0.1 to $0.6 \mathrm{wt}$. \% used in our study is more than a decade higher than the one used in the literature (see for example Arratia et al. 2008, 2009 used PAM with concentrations of 100ppm, and PAA solutions are known to be much more viscoelastic compare to CMC or PEO solutions) - which confer to our system a very special behavior of the droplet in formation.

The mechanisms of evolution of the morphology of polyacrylamide aqueous solutions dispersed phase in the dripping and jetting modes co-flowing with a silicone oil continuous phase in coaxial capillaries were found for different polymer concentrations and continuous Newtonian phase flow rates. In the dripping mode of flow, the liquid filament length linked to the terminal droplet, increases linearly in time due to the droplet's moving away from the inner capillary. The droplet velocity was shown to be constant and does not depend on polymer concentration. In this case, the decrease of the filament diameter is caused by two factors increase of the jet length and flow of polymer solution into the terminal droplet due to contraction of the filament under the capillary pressure.

In the jetting flow mode, the thinning of the liquid thread can be subdivided into three stages. In the first stage, the average jet velocity increases, whereas in the second one the jet motion becomes steady. The transition between the first and second stages of jet propagation corresponds to the point where the jet average velocity approaches the maximum velocity of the continuous medium co-flowing in the external capillary. The transition from the second to the third stage is associated with the first appearance of visible fluctuations on the liquid thread caused by the growing capillary waves resulting in transformation of some of them to beads on a string. The diameter of the filaments between the beads was found to decrease exponentially. The corresponding relaxation times depend mainly on polymer concentration and do not vary with the flow rate of the continuous phase. The filament length between various bead generations decreases in time as a power law function. It was found that in the steady-state jet propagation 
mode, the increase of the beads sizes is related to the outflow of the incompressible polymer solution from the connecting filaments due their capillary compression. In this case, the dimensionless lateral dimensions of filaments and beads evolve in an universal way regardless of the polymer concentration and flow rates of co-flowing continuous medium.

\section{Acknowledgments}

SP and SV are thankful to Arkadii Arinstein for the valuable discussions and grateful to the Russian Foundation for Basic Research (grant nos. 18-29-17072 and 18-53-15013) and partly to the State Assignments 0082-2019-0005 and 0089-2019-0001 of Russian Academy of Sciences for the financial support of this work. CS and DF are grateful to the CNRS for funding through the PRC DroMir project.

\section{References}

1. S. Middleman, Stability of a viscoelastic jet, Chem. Eng. Sci. 20 1037-1040 (1965).

2. M. Goldin, H. Yerushalmi, R. Pfeffer, R. Shinnar, Breakup of a laminar capillary jet of a viscoelastic fluid, J. Fluid Mech. 38 (4) 689-711 (1969)

3. J. W. S. Rayleigh, On the instability of jets, Proc. London Math. Soc. 10, 4-13 (1878).

4. J. W. S. Rayleigh, On the stability of a cylinder of viscous liquid under capillary force, Philos. Mag. 34, 145 (1892).

5. S. Tomotika, On the instability of a cylindrical thread of a viscous liquid surrounded by another viscous fluid, Proc. R. Soc. London, Ser. A 150, 322-337 (1935).

6. J. Eggers, Nonlinear dynamics and breakup of free-surface flows, Rev. Mod. Phys. 69, 865 (1997).

7. D. W. Bousfield, R. Keunings, G. Marrucci and M. M. Denn, Nonlinear analysis of the surface-tension driven breakup of viscoelastic fluid filaments, J. Non-Newtonian Fluid Mech. 21, 79 (1986).

8. M. Renardy, Some comments on the surface-tension driven break-up (or the lack of it) of viscoelastic jets, J. Non-Newtonian Fluid Mech. 51, 97 (1994).

9. M. Renardy, A numerical study of the asymptotic evolution and breakup of Newtonian and viscoelastic jets, J. Non-Newtonian Fluid Mech. 59, 267 (1995).

10. J. Li and M. A. Fontelos, Drop dynamics on the beads-on-string structure for viscoelastic jets: A numerical study, Phys. Fluids 15, 922 (2003).

11. Yarin, A.L. Free liquid jets and films: hydrodynamics and rheology. Longman Sci. \& Tech. with John Wiley \& Sons, Inc., New York, 446 p. (1993).

12. M. S. N. Oliveira and G. H. McKinley. Iterated stretching and multiple beads-on-a-string phenomena in dilute solutions of highly extensible flexible polymers, Physics Fluids 17, 071704 (2005). 
13. C. Wagner, Y. Amarouchene, D. Bonn, and J. Eggers, Droplet Detachment and Satellite Bead Formation in Viscoelastic Fluids, Phys. Rev. Lett. 95, 164504 (2005).

14. C. Clasen, J. Eggers,M.A. Fontelos, J. Li, andG.H.McKinley, The beads-on-string structure of viscoelastic threads, J. Fluid Mech. 556, 283 (2006).

15. R. Sattler, C. Wagner, and J. Eggers, Blistering Pattern and Formation of Nanofibers in Capillary Thinning of Polymer Solutions, Phys. Rev. Lett. 100, 164502 (2008).

16. S. L. Goren and M. Gottlieb, Surface-tension-driven breakup of viscoelastic liquid threads, J. Fluid Mech.120, 245-266 (1982).

17. S. L. Anna and G. H. McKinley, Elasto-capillary thinning and breakup of model elastic liquids. J. Rheol. 45, 115 (2001)

18. M. S.N. Oliveira, R. Yeh, G. H. McKinley, Iterated stretching, extensional rheology and formation of beads-on-a-string structures in polymer solutions. J. Non-Newtonian Fluid Mech. 137, 137-148 (2006).

19. P.P. Bhat, S. Appathurai, M.T. Harris, M. Pasquali, G.H. McKinley, O.A. Basaran Formation of beads-on-a-string structures during break-up of viscoelastic filaments, Nature Physics 6, 625631 (2010).

20. A.Ya. Malkin, A. Arinstein, V.G. Kulichikhin, Polymer extension flows and instabilities. Progress in Polymer Science 39, 959-978 (2014).

21. V. Kulichikhin, Al. Ya. Malkin, A. V. Semakov, I. Skvortsov, A. Arinstein, Liquid filament instability due to stretch-induced phase separation in polymer solutions. Europ. Phys. J. E 37, 9965 (2014).

22. Bazilevskii A.V., Entov V.M., Rozhkov A.N. Breakup of an Oldroyd liquid bridge as a method for testing the rheological properties of polymer solutions // Polymer Science. Ser. A. 43. №. 7. P. 716-726 (2001).

23. A. B. Bazilevskii and A. N. Rozhkov Dynamics of the Capillary Breakup of a Bridge in an Elastic Fluid. Fluid Dynamics, 2015, 50, No. 6, pp. 800-811.

24. A.L. Yarin, B. Pourdeyhimi, S. Ramakrishna Fundamentals and Applications of Micro- and Nanofibers, Cambridge University Press, 2014, 431 pages.

25. R. Sattler, C. Wagner, and J. Eggers, "Blistering pattern and formation of nanofibers in capillary thinning of polymersolutions," Phys. Rev. Lett. 100, 164502 (2008)

26. R. Sattler, S. Gier, J. Eggers, and C. Wagner, The final stages of capillary break-up of polymer solutions. Phys. Fluids 24, 023101 (2012)

27. A. Deblais, 1 K. P. Velikov, 1,2 and D. Bon, Pearling instabilities of a viscoelastic thread Phys. Rev. Lett. 120, 194501 (2018).

28. J. Eggers, Instability of a polymeric thread. Phys. Fluids 26, 033106 (2014)

29. A. V. Semakov, I. Yu. Skvortsov, V. G. Kulichikhin, A. Ya. Malkin, "From capillary to elastic instability of jets of polymeric liquids: Role of the entanglement network of macromolecules", JETP Letters, 101:10 (2015), 690-692.

30. A. V. Subbotin and A. N. Semenov, Multiple droplets formation in ultrathin bridges of rigid rod dispersions. J. Rheology, 64, 13-27 (2020). 
31. A Ya Malkin, A V Subbotin, V G Kulichikhin, Stability of polymer jets in extension: physicochemical and rheological mechanisms, Russ. Chem. Rev., 2020, 89 (8), 811-823.

32. J. W. Hoyt, J. J. Taylor and C. D. Runge, The structure of jets of water and polymer solution in air. J. Fluid Mech., 63635 - 640 (1974).

33. J.J. Cooper-White*, J.E. Fagan, V. Tirtaatmadja, D.R. Lester, D.V. Boger. Drop formation dynamics of constant low-viscosity, elastic fluids J. Non-Newtonian Fluid Mech. 106, 29-59 (2002).

34. V. Tirtaatmadja, G. H. McKinley, J. J. Cooper-White, Drop formation and breakup of low viscosity elastic fluids: Effects of molecular weight and concentration. Phys. Fluids 18, 043101 (2006).

35. J.M.H. Janssen, Dynamics of liquid-liquid mixing. PhD Thesis, Eindhoven University of Technology, 1993.

36. P. E. Arratia, J.P. Gollub and D.J. Durian, Polymeric filament thinning and breakup in microchannels. Phys. Rev. E 77, 036309 (2008).

37. W. Du, T. Fu , Q. Zhang, C. Zhu, Y. Ma, Self-Similar Breakup of Viscoelastic Thread for Droplet Formation in Flow-Focusing Devices. AIChE, 63, 11, 5196-5206 (2017).

38. G. H. McKinley and A. Tripathi, How to extract the Newtonian viscosity from capillary breakup measurements in a filament rheometer" J. Rheol. 44, 653 (2000).

39. V.M. Entov, E.J. Hinch, Effect of a spectrum relaxation times on the capillary thinning of a filament elastic liquids. J. Non-Newtonian Fluid Mech. 72, 31-53 (1997).

40. M.D. Chilcott, J.M. Rallison Creeping flow of dilute polymer solutions past cylinders and spheres // Journal of Non-Newtonian Fluid Mechanics, 29 (1988) 381-432. 